\title{
Activités
}

$14-2$ | 2017

Dimensions et conditions d'une intervention capacitante

\section{L'acceptation de la voiture électrique : un agent transformateur des pratiques socio-domestiques}

Acceptation of the electric car: A transforming agent of socio-domestic practices

Lénaïc Poupon, Chrystèle Philipps-Bertin, Marc-Éric Bobillier Chaumon et Nikos Kalampalikis

\section{OpenEdition}

\section{Journals}

Édition électronique

URL : https://journals.openedition.org/activites/3044

DOI : 10.4000/activites.3044

ISSN : 1765-2723

Éditeur

ARPACT - Association Recherches et Pratiques sur les ACTivités

Référence électronique

Lénaïc Poupon, Chrystèle Philipps-Bertin, Marc-Éric Bobillier Chaumon et Nikos Kalampalikis,

"L'acceptation de la voiture électrique : un agent transformateur des pratiques socio-domestiques », Activités [En ligne], 14-2 | 2017, mis en ligne le 15 octobre 2017, consulté le 21 septembre 2021. URL http://journals.openedition.org/activites/3044 ; DOI : https://doi.org/10.4000/activites.3044

Ce document a été généré automatiquement le 21 septembre 2021.

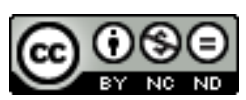

Activités est mis à disposition selon les termes de la licence Creative Commons Attribution - Pas d'Utilisation Commerciale - Pas de Modification 4.0 International. 


\section{L'acceptation de la voiture électrique : un agent transformateur des pratiques socio- domestiques}

Acceptation of the electric car: A transforming agent of socio-domestic practices

Lénaïc Poupon, Chrystèle Philipps-Bertin, Marc-Éric Bobillier Chaumon et Nikos Kalampalikis

\section{NOTE DE L'ÉDITEUR}

Article soumis le 21/11/2016, accepté le 25/07/2017

\section{Introduction et contexte de la recherche}

1 Les transports font partie intégrante de la vie quotidienne. Les déplacements qu'ils permettent sont indispensables à la réalisation de nombreuses activités. Pour les mener à bien, différents modes de transports, motorisés ou non, individuels ou collectifs, sont à disposition des personnes. En 2015 ${ }^{1}$, les véhicules particuliers représentent $82 \%$ du transport de personnes en France. Ce sont à plus de $90 \%$ des voitures à motorisation thermique. Ces dernières sont à l'origine de nuisances environnementales, notamment le bruit et la pollution.

2 Actuellement, la pollution atmosphérique est une préoccupation importante dans notre société du fait de son impact sur la santé humaine et l'environnement. Or les transports routiers représentaient $27 \%$ des émissions de pollution atmosphérique en 2013. Il devient donc essentiel de trouver de nouvelles manières de se déplacer, d'adopter de nouvelles pratiques de transport pour pallier à ces problèmes environnementaux. La voiture 
électrique (rapport ${ }^{2}$ de l'ADEME ${ }^{3}$ ) pourrait contribuer à la réduction de la pollution en France notamment en zone urbaine. Cependant son usage reste marginal, même si actuellement on remarque une progression de ses ventes ${ }^{4}$. Cela nous amène à nous interroger sur les freins à son adoption (qui se réfère à un état où les utilisateurs ont pris la décision d'utiliser une innovation) ou la prise de décision d'utiliser le véhicule et à son usage ou au contraire sur les éléments facilitateurs, en prenant comme cadre d'analyse l'activité socio-domestique et l'expérience de conduite qui peuvent être affectées par l'utilisation de ce type de véhicule.

Dans ce contexte, cet article présente une partie des résultats d'une recherche dont la problématique vise l'étude du processus menant à l'acceptation de la VE. Notre démarche peut être décomposée en trois phases principales. La première se situe avant tout usage et pourra mener ou non à l'utilisation du véhicule. La phase de l'acceptabilité sociale a fait l'objet de nombreux modèles qui tentent d'expliquer et de prédire des intentions d'utilisation à partir notamment de perceptions a priori de la technologie et de son usage. Elle est suivie de la phase d'acceptabilité pratique, lors des premiers temps de l'utilisation. Son étude repose principalement sur des approches ergonomiques et porte sur les qualités instrumentales (utilité, utilisabilité, efficience, etc.) et non instrumentales (émotions, éprouvés subjectifs, etc.) de la technologie. Au-delà de l'adoption de la VE et de ces premières utilisations, dans cet article, nous avons choisi de nous intéresser à un usage à long terme réel et incarné en situation (perspective de l'acceptation située, Bobillier-Chaumon, 2016). Dans cette perspective, notre question de recherche nous conduit à moins nous intéresser aux facteurs d'acceptation des technologies elles-mêmes qu'à l'acceptation des nouvelles pratiques (ou à l'empêchement ou au renoncement des anciennes) liées à l'usage de cet objet technique. Il s'agit de comprendre comment la voiture électrique et les pratiques spécifiques qu'elles réclament viennent s'articuler - ou pas - avec les conditions et les modalités d'exercice des activités de ses usagers. On cherche ainsi à analyser en quoi l'usage de ce nouveau mode de transport entrave ou favorise le rayonnement de l'activité sur différentes dimensions du système sociodomestique. C'est alors pour maintenir son activité à un niveau acceptable que l'individu sera amené à accepter ou à rejeter cet artefact technique.

\section{La prise en compte de l'activité dans l'acceptation de la voiture électrique}

4 L'acceptation porte sur l'analyse des usages réels, et peut être définie par l'intérêt que l'individu trouve à s'approprier une technologie. «L'acceptation se présente comme la façon dont un individu, un collectif, mais aussi une organisation perçoit les enjeux liés aux nouvelles technologies (atouts, bénéfices, risques et opportunités) et y réagissent favorablement ou non. [...] Ainsi l'introduction d'une nouvelle technologie peut engendrer une situation de crise avec de nombreuses ruptures (modifications de la tâche, mode de raisonnement, procédure, organisation de l'action, etc.). En conséquence l'individu va devoir se repositionner dans cette nouvelle configuration ou va écarter la menace en refusant le dispositif » (Bobillier-Chaumon, Dubois, \& Retour, 2006, p. 246).

5 La VE étant utilisée dans les pratiques quotidiennes, elle s'inscrit dans un contexte d'usage sociodomestique. Compte tenu de ses spécificités, son usage (notamment son autonomie limitée et son temps de charge pouvant être important) peut modifier les 
pratiques de déplacement ainsi que leur gestion, engendrer des reconfigurations au niveau des activités déployées par l'utilisateur et de son foyer, mais aussi modifier l'organisation du système social et familial. Il est donc nécessaire de prendre en compte l'activité, mais également l'environnement social dans lequel elle se déploie. Pour mettre en lumière ces possibles reconfigurations et leurs effets sur l'acceptation de la VE, nous allons dans un premier temps nous appuyer sur l'approche instrumentale (Rabardel, 1995), pour identifier les mécanismes à l'œuvre dans la création de nouveaux usages. Enfin, pour comprendre comment ils affectent les activités de déplacements et les interactions sociales, nous appliquerons le modèle des systèmes d'activité (SA) de Engeström (1987), adapté à un contexte sociodomestique (Bobillier-Chaumon, 2013).

\subsection{L'appropriation de la VE à travers la construction de ses usages : l'approche instrumentale}

6 Les voitures électriques (VE) même si elles sont proches des voitures thermiques s'en différencient en de nombreux points que ce soit au niveau de la conduite (similaire aux boites de vitesses automatiques), du réapprovisionnement en énergie (via une prise électrique), ou dans la manière d'appréhender les déplacements en raison de son autonomie et de sa charge. L'appropriation offre un cadre permettant de comprendre comment se créent ou se recréent les usages de la VE en fonction de la technologie, de l'individu et de l'environnement social.

7 Dans cet article, nous nous référons au processus d'appropriation comme étant l'intégration de la technologie par l'individu à son quotidien, en l'adaptant à ses besoins, ses pratiques et ses valeurs (Millerand, 1999 ; Proulx, 2002). Cette intégration dépendrait à la fois des qualités, des performances de la technologie, mais également des significations de l'usage (Mallein \& Toussaint, 1994). Nous retrouvons une idée proche chez Orlikowski (1991) pour qui l'appropriation passe par l'attribution par les utilisateurs d'un sens à la technologie. Il existe donc une dualité dans la construction des technologies : d'un côté, elle est physiquement construite par les concepteurs, et de l'autre, elle est individuellement et socialement construite par les utilisateurs à travers leur usage et les significations données à la technologie. Ce sont l'usage, l'activité et le système social où se déploie cet usage et l'appropriation qui en découle qui vont donner un sens et sa valeur à la technologie. Cette dernière n'étant pas statique, elle peut potentiellement changer au cours de son existence en ayant d'autres propriétés et modalités d'usage. Pour Carroll, Howard, Peck, \& Murphy (2003) l'appropriation renvoie à la manière d'évaluer, d'adopter, d'adapter et d'intégrer la technologie dans ses pratiques quotidiennes. Les utilisateurs vont sélectionner certains composants de la technologie, l'adapter, selon leurs besoins jusqu'à la stabilisation des usages.

8 La manière dont se construisent les usages de la VE peut être appréhendée grâce à l'approche instrumentale (Rabardel, 1995). Cette dernière s'intéresse à la fois aux ajustements que l'acteur opère sur l'artefact ainsi qu'aux transformations de sa propre activité en vue d'intégrer cet artefact. Cette approche comporte l'avantage de se situer du point de vue de l'utilisateur, de prendre en compte sa subjectivité. Ainsi, nous pouvons donc étudier la relation d'usage à partir des rapports entre l'instrument (la VE) et le sujet dans l'action (la conduite). La notion d'instrument (Rabardel, 1995) désigne un artefact en situation. Il y a donc un rapport instrument, sujet (l'utilisateur), objet (vers lequel l'action est dirigée p.ex. la réalisation d'un déplacement). Dans ce sens, l'instrument devient un 
moyen d'action pour le sujet. Le rapport à l'artefact est donc appréhendé du point de vue du sujet, de son activité, de son action. Un instrument comprend un artefact et des schèmes d'utilisation qui permettent au sujet d'assimiler les situations et les objets auxquels il est confronté. Un même schème d'utilisation peut s'appliquer à une multitude d'artefacts de la même classe ou de classe voisine à l'instar de certaines tâches de conduite communes aux voitures thermiques et électriques. Inversement, de nombreux schèmes d'utilisation peuvent être appliqués à un artefact, lui attribuant des significations et fonctions différentes. Les évolutions des schèmes et du sujet sont liées à deux processus complémentaires (Rabardel, 1995). (i) L'assimilation: une conduite donnant lieu à répétition va se schématiser. Le schème va donc être l'ensemble structuré des caractères généralisables de l'action. Il va donc permettre la répétition de l'action à un autre contenu, à un autre artefact. (ii) L'accommodation : quand l'individu éprouve des difficultés à assimiler de nouveaux artefacts avec les schèmes préexistants, il va devoir les accommoder. Cela va donner lieu à des tâtonnements, pour arriver à des transformations et à la création de nouveaux schèmes pouvant amener à une genèse instrumentale (Rabardel, 1995).

9 La création des usages est un processus mouvant, qui s'inscrit dans différentes temporalités : celle de l'activité qui peut être brève et celle plus longue de la vie des individus (Nogry, Wagner \& Decortis, 2015). Selon l'approche de Rabardel (1995), l'appropriation va correspondre à une ou plusieurs genèses instrumentales. Cette dernière fait référence à l'émergence d'instrument pour le sujet, qui a deux orientations : avec l'instrumentation, l'artefact avec ses contraintes et possibilités va influencer les actions de l'individu. Il peut ainsi faire émerger de nouveaux schèmes d'action ou d'utilisation pour l'usage le plus pertinent de l'artefact. Ceux-ci pourront être ensuite reproduits sur d'autres artefacts. L'instrumentalisation désigne l'adaptation de l'artefact par l'individu à ses besoins et à sa logique d'usage. Il s'appuie sur les propriétés intrinsèques de l'artefact pour faire émerger de nouvelles fonctions, qu'elles soient ponctuelles ou durables.

10 La construction de l'usage et des pratiques de conduite des VE doit être envisagée à l'aune du contexte dans lequel l'activité se déploie, qu'il soit physique ou social (Licoppe, 2008; Relieu, Salembier, \& Theureau, 2004; Theureau, 2004). C'est dans la situation et en fonction de celle-ci que vont se créer ses usages, eux même à la source de transformations dans les activités de déplacements. En effet, l'action des sujets se construit en fonction des circonstances, des opportunités et des contraintes du contexte (Suchman, 1987). Elle n'est donc pas déterminée à l'avance, rationalisée selon un plan que l'individu a prévu et qu'il met en œuvre, mais par les caractéristiques de la situation, guidée par un but. Ce qui est en jeu dans l'étude de l'acceptation de la VE n'est pas seulement l'adoption d'un nouveau type de véhicule, mais aussi les transformations, les reconfigurations qu'il peut engendrer. Nous pouvons à nouveau faire ici un rapprochement avec la notion de genèse instrumentale (Rabardel, 1995). La VE vient s'intégrer dans une activité (déplacement) pour laquelle une technologie existe et est utilisée communément : la voiture thermique. L'appropriation de la voiture électrique, pourrait donc se faire à travers des transferts d'usage entre ces deux technologies proches, par une réutilisation ou non de schèmes existants, la création de nouveaux, voire la transformation d'anciens. La genèse instrumentale établit que le sujet transforme, instrumentalise la technologie pour en faire quelque chose d'autre (notions de détournement, de contournement, de déplacement d'usage, de catachrèse...) en fonction de la situation et de ses finalités; ce 
qui pourrait finalement déterminer de nouveaux usages. Ces derniers pourraient être considérés comme de nouveaux buts émergeant en situation, en fonction du contexte, et orientant l'action.

\subsection{Situer les activités d'usage de la VE}

11 L'approche des systèmes d'activité (SA) de Engeström (1987) nous offre des outils pour étudier l'activité dans son contexte réel, situer l'activité, re-contextualiser les usages de la VE. Elle permet d'appréhender l'environnement dans lequel se déroulent les activités de déplacements et la manière dont il les influence. Elle vise à comprendre comment l'activité se développe, et comment les individus qui la réalisent se développent euxmêmes.

12 La théorie de l'activité est issue de l'école russe de la psychologie du développement humain et a été initiée par Vygotsky (1985). Centrée autour du concept de médiation elle montre comment les artefacts médiatisent les activités humaines. L'instrument n'est donc pas un simple objet avec des propriétés spécifiques, mais un objet social, avec des modalités d'emplois développées au cours du temps à travers des interactions collectives (Rabardel, 1995).

13 Avec l'approche des systèmes d'activité (SA) (Figure 1), Engeström (1987) étend les théories de l'activité qui se voient appliquées au fonctionnement de systèmes d'activité collective, médiatisée par l'artefact et orientée vers l'objet. Pour comprendre et analyser les médiations au sein d'un SA, cet auteur part de la triade sujet, objet, instrument, développée par Vygotsky (1985) et l'étend aux règles, à la communauté, et à la division des tâches. Chacun de ces éléments représente un pôle du système. Les relations entre ces pôles permettent de se figurer les médiations, les tensions, les contradictions, qui vont participer au développement de l'activité (Licoppe, 2008).

Mobiliser le modèle du SA appliqué à l'analyse des situations d'usage de la VE dans un contexte sociodomestique présente deux intérêts. Le premier est d'appréhender l'activité dans sa globalité et de repérer les contradictions intra-SA. Elles peuvent exister à la fois au sein d'un pôle (niveau primaire) et entre les pôles du modèle (niveau secondaire). Le second est de mettre en lumière comment se transforme l'activité. Plus précisément, cela permet de comprendre comment l'usager va dépasser ces contradictions et trouver «les éléments qui nourrissent une nouvelle manière de penser son activité » avec ce dispositif de transport (Bonneau, 2010, p. 103). 
Figure 1 : Structure d'un système d'activité (Engeström, 1987, p. 78).

Figure 1: Structure of an activity system (Engeström, 1987, p. 78)

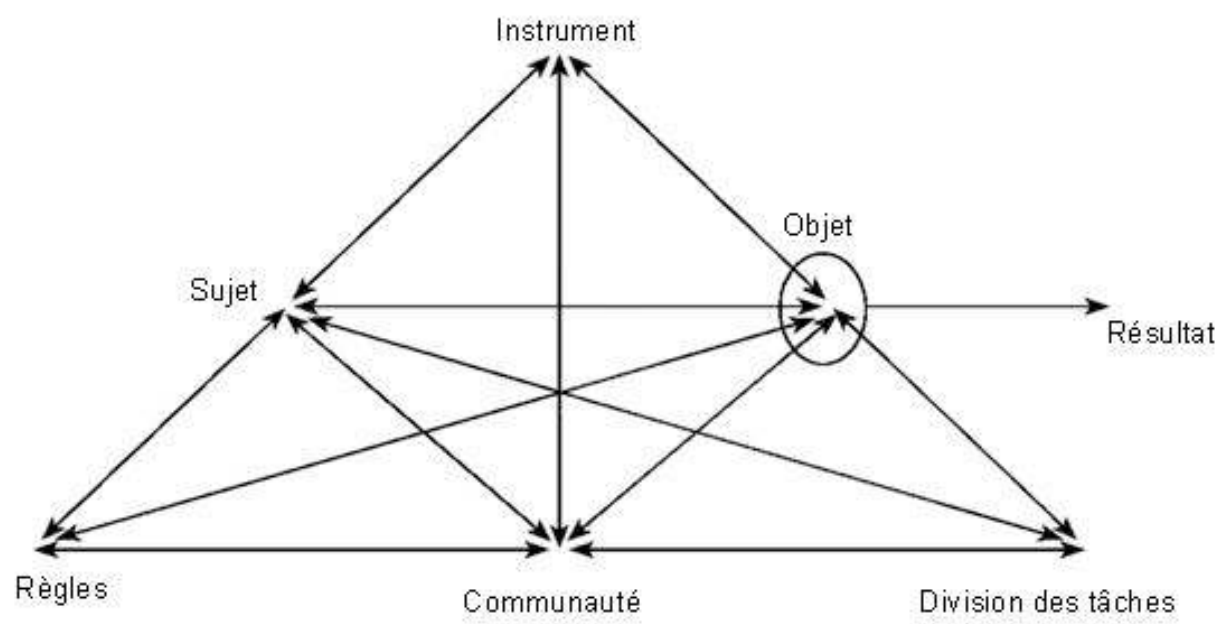

Nous retrouvons dans ce modèle (Figure 1) le triangle de Vygotsky ou le rapport du sujet à l'objet est médiatisé par l'instrument entrainant une activité. Engeström a ajouté les pôles "règles", "communauté » et "division des tâches". Ainsi il met en avant le caractère socialement ancré de la réalisation d'une activité, à travers des règles au sein d'une communauté où l'ensemble des acteurs se répartit des tâches en vue de la réalisation d'un objectif commun. L'activité connait donc un développement dynamique. Un système d'activité intègre :

- Un sujet qui peut être un individu ou un sous-groupe en fonction du point de vue adopté.

- Un objet vers lequel l'activité est dirigée.

- Un instrument tel qu'un outil, un objet matériel ou encore des signes ou des symboles.

- Une communauté qui comprend des individus ou groupes partageant le même objet général.

- Des règles qui font référence aux régulations, aux normes et aux conventions implicites et explicites qui limitent les actions et interactions au sein du SA.

- La division des tâches qui fait référence à la division horizontale entre les membres de la communauté et la division verticale du pouvoir et des statuts.

16 Nous pensons que ce modèle offre un cadre pour appréhender le SA domestique, à savoir les interactions au sein de la structure familiale, les ajustements sociaux entre les membres, le développement des activités et des usages en lien avec la VE. Cette approche permet de situer l'acceptation dans un processus dynamique qui se développe à la fois de manière sociale à travers des interactions et des conflits, mais également de façon temporelle.

Bien que développé pour l'étude de l'activité dans un cadre professionnel, il nous semble pertinent d'appliquer les éléments du modèle d'Engeström (1987) à un cadre sociodomestique (Bobillier-Chaumon, Cuvillier, Durif-Bruckert, Cros, Vanhille, \& Bekkadja, 2014) et donc au cas de la VE. En effet, les activités de déplacements sont médiatisées par la VE dans un contexte familial et orientées vers un but. Ce système inclut une communauté composée des différents membres du foyer (parents, enfants...), il a donc une orientation collective, où chaque acteur peut avoir des buts différents (se rendre en des lieux différents), des intérêts ou des points de vue différents (économique, écologique, etc.). De plus, les domaines de vie familiale et professionnelle et leurs 
systèmes d'activité respectifs évoluent au cours du temps (début de la vie professionnelle, changement de travail, naissance, etc.) tout comme les pratiques et modalités de déplacement. Les activités de déplacement doivent donc être appréhendées en fonction à la fois de l'histoire du foyer, et du rapport à l'automobile ainsi qu'aux déplacements.

Ce modèle permet également de rendre compte des contradictions qui émergent de ce SA et qui peuvent notamment témoigner «d'un manque de compatibilité à l'intérieur des éléments, entre les éléments, entre différentes activités et entre différentes phases d'une activité " (Kuuti, 1996, p. 34). Dans le cadre des activités sociodomestiques, ces contradictions se manifestent comme des perturbations qui interrompent le flux normal de l'activité. La compréhension de ces tensions se révèle donc essentielle dans notre recherche afin de comprendre les difficultés des utilisateurs lorsqu'ils doivent intégrer une VE dans leurs pratiques de vie courante.

\subsection{Le système d'activité appliqué à un contexte d'usage domestique}

19 Afin d'appliquer le modèle des systèmes d'activité à un cadre domestique, il faut au préalable saisir ce contexte particulier. Nous allons apporter ici des éléments pour la compréhension des activités réalisées dans ce cadre spécifique. Nous allons plus particulièrement nous intéresser à la façon dont certaines pratiques de l'espace domestique se forment, se développent ou se dégradent sous l'effet de nouveaux artefacts et à quelles et sous quelles conditions ces reconfigurations restent acceptables.

Schatzki (1996) propose une approche par les pratiques sociales à partir de l'étude de «l'espace social» ou des lieux de consommation et de transformation de la vie sociale (Dubuisson-Quellier \& Plessz, 2013). Les pratiques sociales sont au cœur de l'analyse : ces dernières auraient la capacité de " recruter des adeptes ». " Une "pratique" est un type de comportement "routinisé" qui consiste en plusieurs éléments interconnectés entre eux : des formes d'activités mentales, des "choses" et leur usage, des connaissances de base constituées de compréhension, savoir-faire, états émotionnels de motivation » (Reckwitz, 2002, p. 249). Shove, Pantzar et Watson (2012), pour définir ce concept de pratique, le décomposent en trois catégories: les éléments matériels (e.g. entités physiques), les compétences (e.g. connaissances procédurales) et les significations (e.g. connaissances déclaratives). Les liens entre ces composants vont participer à l'évolution des pratiques.

Certaines pratiques coexistent et sont co-localisées, ainsi elles peuvent «s'emboiter » les unes aux autres pour composer des « practice bundle » ou paquets de pratiques. Dans les relations intra-pratiques complexes, il peut également exister des phénomènes de concurrence ou de coopération (Shove, Pantzar \& Watson, 2012). En fonction des contraintes temporelles, spatiales, mais également sociales, toutes les pratiques ne pourront être réalisées, un choix devra donc s'opérer. Selon Shove Pantzar et Watson (2012), ce sont les pratiques qui vont façonner la dimension temporelle plutôt que l'inverse. En raison de leurs aspects récurrents, elles sont susceptibles de créer des formes de routinisation et ainsi elles vont donc transformer du temps libre en temps contraint. Cette temporalité va être plus ou moins contrainte en fonction des nécessités de coordination qui s'imposent à l'individu, mais aussi en fonction de certaines normes sociales qui vont rendre acceptable ou même parfois requérir de passer plus de temps sur une activité que sur une autre (Dubuisson-Quellier \& Plessz, 2013). 
22 L'émergence d'une acceptation de la VE dans un contexte d'usage sociodomestique nécessite donc une convergence intra-pratiques. C'est-à-dire que les pratiques liées à la VE devront être compatibles, s'intégrer dans les pratiques domestiques déjà en place avec la voiture thermique (par son remplacement ou leur coexistence) et le rythme qu'elles imposent. Il ne faut pas que les nouvelles pratiques rentrent en concurrence avec les pratiques quotidiennes et nuisent ou empêchent leur exécution. Il faut qu'elles assurent leur réalisation ou favorisent et développent leur déroulement. Plus largement, des ajustements dans le système d'activités domestique de la famille s'effectueront donc avec l'introduction de la VE.

La prise en compte du contexte sociodomestique dans lequel va s'insérer la VE nécessite de considérer des interactions, des négociations au sein du foyer, avec des conflits ou des tensions, aussi bien que des consensus. Tout comme l'approche par la pratique sociale, la domestication (Silverstone \& Haddon, 1996) s'intéresse à l'intégration d'une technologie dans une routine quotidienne et ses conséquences que ce soit pour un adoptant individuel ou collectif (un foyer, un groupe social, etc.). L'approche de la domestication aura cependant un point de vue plus large, elle « cherche à évaluer les processus qui façonnent l'adoption et l'utilisation des TIC, mais ce faisant s'interrogent également sur ce que les technologies et services signifient pour les individus, comment ils vivent leurs usages des TIC et quel rôle ces technologies viennent jouer dans leurs vies » (Haddon, 2011, p. 312).

Le processus de domestication commence avant l'acquisition et se poursuit durant l'usage avec l'intégration de la technologie dans la vie quotidienne. Après la phase d'acquisition de la technologie, suit une phase d'expérimentation au cours de laquelle des modes d'utilisation routiniers s'installent. Cependant à plus long terme, l'usage continue d'évoluer grâce à la dynamique du changement technologique, des changements sociaux ou des changements au sein du foyer (Haddon, 2011). La domestication n'est donc pas statique, elle ne s'arrête pas à l'achat de la technologie, il s'effectue des ajustements tout au long des usages. Ils sont liés aux circonstances de la vie quotidienne, aux interactions avec autrui.

25 La domestication nous permettra de comprendre le mouvement d'intégration de la VE dans la vie quotidienne des utilisateurs : quelle signification elle peut avoir pour eux, ce qu'elle représente en termes de valeurs personnelles et d'image de soi, comment ils vivent les usages, comment sont reformulées leurs vies autour des possibilités qu'elle offre, ou des contraintes qu'elle impose.

\subsection{Questions de recherche}

Nous cherchons à appréhender le maintien de l'usage de la VE dans une temporalité relativement longue. Nous nous intéressons à la manière dont elle peut être introduite dans un foyer en remplacement d'une voiture thermique, comment elle s'intègre dans cet environnement sociodomestique et les pratiques des utilisateurs.

Les VE possédant une autonomie limitée, avec un temps de charge pouvant être important, elles requièrent de nouvelles tâches de planification et d'organisation des déplacements. Ces contraintes pourraient de plus avoir un effet sur les activités de conduites. Il s'agit donc pour nous d'appréhender les nouveaux usages créés par l'utilisation d'une VE. En effet, la création de ces nouveaux usages contribue à son appropriation et participe à son acceptation. 

s'inscrit et affecte les domaines de vie: social, domestique, professionnel et familial. Il pourrait donc modifier l'organisation du SA (Engeström, 1987) et les différentes pratiques qui en résultent (Schatzki, 1996); ou bien encore, affecter les rapports interpersonnels au sein de la structure sociale et familiale (Silverstone \& Haddon, 1996). Par exemple, son autonomie réduite pourrait empêcher certains déplacements imprévus, ou simplement trop longs, et donc affecter d'autres activités en lien avec les déplacements comme les loisirs.

Nous cherchons plus précisément à identifier les incidences que peut avoir l'usage de la VE sur les différentes dimensions du système d'activité socio-domestique qui composent le foyer. En d'autres termes, est-ce que les interactions, les règles de vie domestique et sociale, la coordination et la répartition/division de l'activité avec d'autres individus sont affectées favorablement ou non par l'usage d'une voiture électrique. On supposera qu'une inadéquation entre d'une part, les pratiques exigées ou suscitées par la VE et d'autre part, les pratiques établies au sein du foyer et/ou acquises avec la voiture thermique conduiraient à un rejet du véhicule. C'est alors pour maintenir ses activités à un niveau acceptable que l'individu sera amené à accepter ou à rejeter ce nouvel artefact technique qu'est la VE. L'enjeu n'est dès lors pas tant que ce nouveau véhicule soit perçu favorablement - comme le mesurent les modèles sociocognitifs de l'acceptabilité sociale -, mais qu'il ait effectivement un effet favorable et durable sur l'individu et sur son système d'activité domestique, en soutenant le déroulement de ses habitudes et de règles de vie et en lui permettant de les déployer à l'échelle de ses différents domaines de vie (famille, loisir, social, professionnel...).

\section{Méthodologie de recherche}

Pour appréhender l'acceptation de la VE, notre objectif était d'être au plus proche de la réalité de son usage dans un contexte sociodomestique. Nous avons donc développé un protocole de recueil de données auprès de particuliers possédant et conduisant une VE au quotidien (déplacements pour les loisirs, déplacements pendulaires, etc.). Le recrutement a été fait principalement via internet en contactant des individus désireux de vendre leur VE. Au vu de la faible taille de la population concernée par notre recherche et des difficultés de rentrer en contact avec des sujets potentiels, nous avons opté pour une méthode qualitative avec plusieurs techniques d'entretien. Nous voulions ainsi maximiser le recueil de données obtenues auprès de chaque participant.

\subsection{Population}

31

Nous avons interrogé 9 individus (Tableau 1), tous possesseurs et conducteurs d'une VE (10 individus au départ, un ayant abandonné entre les deux entretiens). Nous avons une majorité de sujets masculins ( 7 hommes et 2 femmes), avec une moyenne d'âge de 45 ans (le plus jeune ayant 26 ans et le plus âgé 65 ans), et une expérience de conduite de 25 ans en moyenne (l'expérience minimum étant de 10 ans et maximum de 47 ans). 
Tableau 1 : Récapitulatif des participants usagers de voiture électrique. Table 1: Summary of electric car users participants

\begin{tabular}{|c|c|c|c|c|l|}
\hline Sujet & âge & Sexe & $\begin{array}{c}\text { expérience conduite } \\
\text { en année }\end{array}$ & $\begin{array}{c}\text { durée de possession VE } \\
\text { en année }\end{array}$ & Modèle VE possédé \\
\hline S1 & 36 & $\mathrm{~F}$ & 17 & 2 & Peugeot Ion \\
\hline S2 & 39 & $\mathrm{M}$ & 20 & 5 & Peugeot 106 électrique \\
\hline S4 & 65 & $\mathrm{M}$ & 47 & 2 & Nissan Leaf \\
\hline S5 & 28 & $\mathrm{M}$ & 10 & 10 & Citroën Saxo électrique \\
\hline S6 & 47 & $\mathrm{~F}$ & 27 & 1,5 & Renault Zoé \\
\hline S7 & 49 & $\mathrm{M}$ & 31 & 1 & Citroën C-Zéro \\
\hline S8 & 38 & $\mathrm{M}$ & 17 & 2 & Renault Zoé \\
\hline S9 & 31 & $\mathrm{M}$ & 18 & 2 & Nissan LEAF \\
\hline S10 & 53 & $\mathrm{M}$ & 35 & 2,9 & Renault Fluence Ze \\
\hline Moyenne & 43 & & 25 & & \\
\hline
\end{tabular}

Pour 2 ménages (S6, S7) un enfant vit au sein du foyer et est susceptible d'utiliser le véhicule. Pour 5 ménages (S1, S2, S4, S8, S9), il y a seulement le couple qui utilise la voiture électrique. Pour un foyer, le véhicule est utilisé par le possesseur (S5) ainsi que son père. Dans le dernier foyer, le possesseur (S10) seul utilise le véhicule électrique.

Tous les conducteurs ayant participé aux entretiens possèdent un second véhicule de type thermique. Tous les foyers sont donc multimotorisés et disposent d'un emplacement privatif avec accès à une prise électrique pour charger leur véhicule (8 habitent dans une maison, et un dans un appartement).

\subsection{Recueil de données, le couplage de différentes techniques d'entretien}

Les entretiens se sont déroulés en deux phases séparées par un délai moyen d'une semaine. Ce délai a été déterminé de manière à être suffisant pour permettre un premier traitement des données, mais en même temps pas trop long pour éviter des expériences, point de vue, modifications d'usage trop importantes entre les deux phases.

Lors de la première phase de l'entretien (d'une durée moyenne de 31 minutes) étaient abordés les usages de la VE de manière ouverte et générale (description de la VE, motivation d'achat, prise en main et usages). Cet entretien était ensuite intégralement retranscrit et faisait l'objet d'une première analyse afin d'identifier les éléments à expliciter lors de la seconde session d'entretien.

$\mathrm{Au}$ cours du deuxième entretien (d'une durée moyenne de 1heure et 11 minutes), nous revenions sur ce qui avait été dit lors du premier pour l'approfondir quand cela était nécessaire. De plus, nous évoquions de nouvelles thématiques notamment la gestion des déplacements, de l'autonomie, l'affectation du véhicule, les comportements de conduite. C'est principalement dans cet entretien que nous avons introduit la méthode des incidents critiques ainsi que des relances inspirées de la technique des entretiens d'explicitation.

Ainsi, pour sélectionner certains aspects des expériences d'usage de la VE en vue de les approfondir, nous avons choisi d'utiliser la méthode des incidents critiques (Flanagan, 1954). Elle incite dans un premier temps le sujet à se remémorer l'incident dans lequel il a été impliqué, puis dans un second temps, avec une série de questions l'invite à expliciter et approfondir son déroulement (Clot, \& Leplat, 2005). Elle permet donc de revenir sur des évènements, des épisodes particuliers vécus par le sujet. Il les décrit en détail, ce qui permet de déterminer, de son point de vue, quelles en sont les causes, les modalités ainsi 
que les conséquences, comment il a réagi par rapport à cet évènement, ce qu'il a mis en place, et les changements de comportement qui ont pu en découler. Malgré la dénomination » incident critique », cette méthode n'est pas centrée sur le négatif, elle cherche à faire identifier par les individus des incidents spécifiques de comportement efficace ou inefficace dans un contexte donné. En effet, un incident critique fait référence à une situation significative pour le sujet, chargée émotionnellement et qui va être à l'origine d'un nouveau comportement. Ainsi, ces incidents pourraient être source de rejet ou d'intégration de la technologie, ou de développement de nouveaux usages.

Afin d'amener les sujets à décrire finement certains aspects des expériences d'usage de la VE, nous avons également utilisé la méthode des entretiens d'explicitation. Ils permettent de «re-situer» le sujet dans le contexte d'activité (Cahour, Brassac, Vermersch, Bouraouis, Pachoud, \& Salembier, 2007), de tenter de rendre plus vivace le souvenir et en même temps de limiter la rationalisation dès les reconstitutions pouvant altérer le souvenir. Lors de nos entretiens, nous avons donc utilisé des relances inspirées de la méthode des entretiens d'explicitations (Vermersch, 1994). Elles visent à positionner l'interviewer dans une posture de remémoration du vécu, qu'il soit cognitif, affectif ou corporel (Cahour, et al., 2007). L'intérêt dans le cadre de notre recherche de ce type de relance, était de favoriser la verbalisation des actions passées réalisées par le sujet, des expériences d'usage de la VE à travers la formulation du vécu, de l'action, y compris celles ayant une dimension pré-réfléchie, ou implicite. En d'autres termes, cette technique permet de favoriser la verbalisation de l'activité quand il est notamment impossible d'en avoir des traces.

\subsection{Méthode d'analyse des données}

Bien que nous ayons conduit deux entretiens, ils ont été pensés et traités comme un tout dans la mesure où le second entretien permettait de préciser certaines dimensions du premier. Nous disposions donc d'un corpus de 9 retranscriptions. Pour examiner ce corpus, nous avons choisi de réaliser une analyse thématique de contenu (Bardin, 2007). Le codage et l'analyse de nos données ont été réalisés avec le logiciel NVIVO 10. Ce dernier aide à l'organisation des données dans des ensembles et sous-ensembles thématiques, ce qui facilite l'analyse et l'interprétation des données. Les principales thématiques étant : la description de la VE, l'achat de la VE, la prise en main de la VE (au début et aujourd'hui), les usages de la VE, la gestion des déplacements, le mode d'affectation de la voiture, la gestion de l'autonomie, les comportements de conduite, les réponses émotionnelles liées à l'usage de la $\mathrm{VE}$, autrui, les incidents rencontrés, le maintien ou non de l'usage de la VE. Dans un premier temps nous avons mené une analyse de chaque transcription de manière indépendante. Chaque entretien a été codé de manière indépendante en reportant systématiquement des extraits du discours des sujets dans des catégories thématiques (nœuds) que nous avons hiérarchisées. Dans un second temps, nous avons réalisé une analyse transversale de notre corpus. Nous avons confronté les différents nœuds, les regroupant ou les divisant pour arriver à une structure hiérarchisée du discours des entretiens, facilitant ainsi son analyse thématique. 


\section{Résultats : Les effets de l'usage d'une voiture électrique sur les activités liées aux déplacements}

\subsection{Construction des usages de la voiture électrique}

L'appropriation d'une VE par les individus implique de faire face à des situations nouvelles induites par des caractéristiques (autonomie, absence de bruit moteur) propres à ce type de véhicule.

\subsubsection{Repenser les déplacements pour faire face à l'incertitude}

41 La VE a une autonomie limitée du fait de l'énergie employée et de son système de recharge. Elle est donc susceptible d'avoir un impact important sur les activités liées aux déplacements, l'organisation domestique, mais également sur les éprouvés subjectifs pendant la conduite. Comme l'ont évoqué les conducteurs, elle peut se révéler source d'incertitude quant à la faisabilité d'un déplacement et générer du stress pendant la conduite. Les conducteurs vont donc mettre en place un certain nombre de stratégies pour gérer au mieux l'autonomie de leur véhicule.

Pour réduire l'incertitude quant à la réalisation de leurs déplacements et activités, tous les sujets ont déterminé l'autonomie " réelle » à partir de leur expérience de conduite, et des informations données par les instruments de bord du véhicule (estimation de l'autonomie restante et indication de consommation d'énergie en temps réel). Ils ont ainsi observé des différences plus ou moins importantes par rapport à l'autonomie annoncée par les constructeurs (de 20 à $100 \mathrm{~km}$ ). Les sujets attribuent ces écarts à une surestimation de la part des constructeurs et à plusieurs facteurs, déterminés à partir de leur expérience de conduite, qui influencent la consommation énergétique du véhicule (Figure 2). À titre d'exemple, le relief va impacter l'autonomie. En montée la VE consomme beaucoup d'énergie, contrairement aux descentes où il consomme peu et où il peut $\mathrm{y}$ avoir un phénomène de rechargement des batteries. Une température très basse peut réduire l'autonomie des batteries. De même l'utilisation d'instruments de bord tels que la radio, ou la climatisation va consommer de l'énergie issue des batteries de la VE et réduire son autonomie. Selon les participants, ces différences participent à la création d'incertitude quant à la fiabilité de la technologie.

Figure 2 : Facteurs réduisant l'autonomie de la voiture électrique.

Figure 2: Factors reducing the autonomy of the electric car

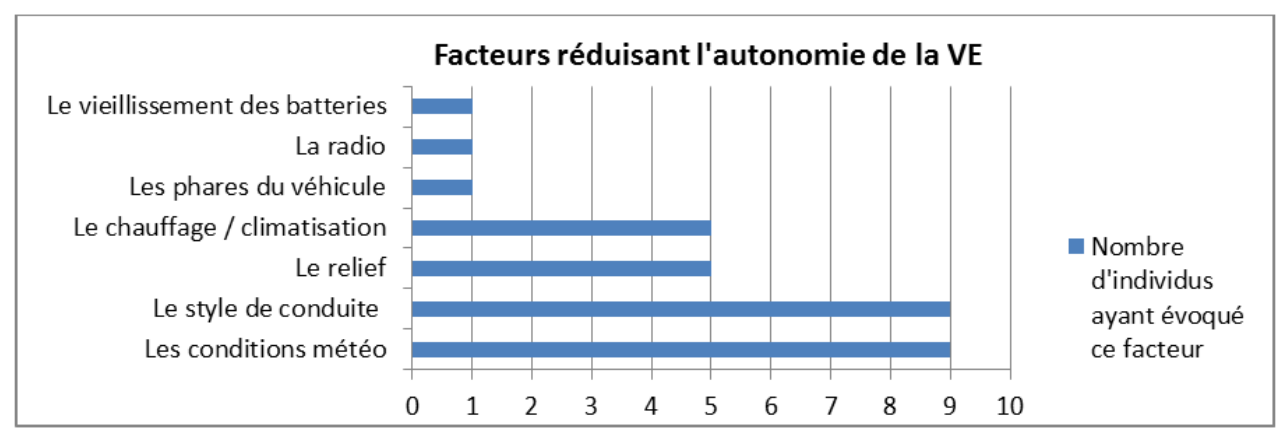


stratégie singulière a été mise en place par 5 individus. Celle-ci consiste en un test d'autonomie. En d'autres termes, le conducteur va volontairement utiliser son véhicule jusqu'à vider complètement ou quasi-complètement ses réserves d'énergie et ainsi évaluer son autonomie réelle.

«J'ai testé 2 ou 3 fois, ces fameux 4 kilomètres restant sur le mode économique et là-dessus, vous avez 4 kilomètres encore. Je l'ai fait deux fois pour être sûr. Vous savez comment j'ai fait ça pour éviter que je me retrouve bloqué, j'ai tourné autour de mon lotissement jusqu'à la dernière petite énergie qui me reste et en fin de compte j'arrivais tout juste à mon garage. Voilà, comment j'ai fait pour vraiment arriver au bout parce que sinon, elle est immobilisée. »(S10).

Ce test vise à vérifier l'autonomie du véhicule et à anticiper sa capacité maximum de déplacements. Il peut donc être considéré comme une stratégie de coping (Lazarus \& Folkman, 1984), ce qui fait référence aux ressources, dispositions personnelles et modalités comportementales mises en place par les individus en réponse aux situations de stress. Ce lien entre la gestion de l'autonomie et les affects (le stress généré par l'incertitude) a été mis en lumière par Nguyen (2013).

Ainsi, les stratégies participent à la création des usages et à l'appropriation de la VE telles que les conçoit Proulx (2002). Elles contribuent à la maitrise cognitive de la technologie à travers la connaissance de l'autonomie. Elles vont amener les conducteurs à la maitrise technique de leur véhicule, témoignant d'une appropriation de celui-ci. Ces stratégies ont été identifiées notamment à l'aide de la méthode des incidents critiques.

Quatre types de stratégies ont été mises en place par les sujets pour gérer leur autonomie (Tableau 2). Chacune est composée d'un ensemble de pratiques ayant pour but d'anticiper leur besoin et d'optimiser l'autonomie.

Tableau2: Type de stratégie de gestion de l'autonomie. Table 2: Type of autonomy management strategy

\begin{tabular}{|c|c|}
\hline Type de stratégie & $\begin{array}{c}\text { Nombre d'individus déclarant } \\
\text { utiliser cette stratégie }\end{array}$ \\
\hline Gestion des déplacements & 9 \\
\hline $\begin{array}{c}\text { Utilisation des informations } \\
\text { des instruments de bord }\end{array}$ & 5 \\
\hline Gestion des éléments de confort & 9 \\
\hline Éco-conduite & \\
\hline
\end{tabular}

Des actions peuvent être réalisées en amont des déplacements dans le but de s'assurer de leur faisabilité grâce à l'optimisation de la gestion des déplacements (9 sujets). Celle-ci consiste en la planification (9) qui peut passer par la prise d'informations sur le trajet a réalisé, la modification d'itinéraires (4) avec par exemple l'évitement de montées, ou la mutualisation des déplacements (1) en profitant d'un même trajet pour se rendre à plusieurs endroits ou réalisé plusieurs activités. Ce sont donc des compétences que les sujets doivent développer, car non nécessaires avec leur voiture thermique. Elles donnent lieu à la création de nouvelles activités et donc de temps contraint (Dubuisson-Quellier \& Plessz, 2013). Cette gestion des déplacements intervient principalement pendant les premiers temps de l'usage. Avec l'expérience, les sujets ne ressentent plus le besoin de planifier leurs déplacements habituels. Quand ceux-ci sont « routinisés » (Reckwitz, 2002), 
les individus réalisent une évaluation rapide de la possibilité de réaliser ou non le trajet. La planification est alors réservée aux déplacements inhabituels, en d'autres termes, à ceux qu'ils ne sont pas sûrs de pouvoir effectuer. L'inquiétude qu'ils peuvent générer (Wellings, Binnersley, Robertson, \& Khan, 2011) tend à disparaitre avec le temps et l'expérience des sujets.

L'expérience des sujets relative à la consommation énergétique de leur véhicule et à la gestion des déplacements a pu être développée avec l'utilisation des informations des instruments de bord du véhicule (7). Ils sont un support cognitif pour la prise de décision et la réalisation de leurs activités. Les principaux sont : l'indicateur du niveau de charge restante (5), l'indicateur de consommation en temps réel (5) et l'ordinateur de bord (1). Ils permettent une maitrise cognitive et technique du dispositif et engendrent la création, ici, de nouveaux styles de conduite en vue d'adapter la technologie à des besoins pratiques.

49 La maitrise de l'autonomie peut passer par la gestion de l'utilisation d'éléments de confort (5). Cela comprend la non-utilisation de la radio (1), de la climatisation (3) ou la restriction de l'utilisation du chauffage (3). Les conducteurs peuvent juger préférable de ne pas les utiliser pour réduire la consommation énergétique. Ils effectuent donc un arbitrage entre leur besoin en autonomie et leur confort. Ce dernier pouvant être plus ou moins pressant selon la situation (p. ex. canicule, grand froid). Cette nécessité de réaliser un compromis pourrait être une source de rejet, car il implique la volonté de la part de l'individu de renoncer à un certain niveau de confort.

50 Finalement, une des stratégies les plus importantes mises en place par les individus pour maitriser leur consommation d'énergie est le développement d'une éco-conduite (9). Ce style de conduite a été développé par la plupart des sujets avec la VE. Il peut être imputé à la nécessité d'optimiser l'autonomie, pour adapter la technologie à leurs besoins, leurs activités. L'éco-conduite renvoie à l'adoption d'une conduite économe en énergie, écologique et économique (Smokers \& Kampman, 2006). Elle implique le développement de nouveaux schèmes d'usage et d'action instrumentée (Rabardel, 1995): pas d'accélération (8) ni de freinages brutaux (7), utilisation du freinage récupératif (7), une conduite « douce » (4) et le maintien d'une vitesse stable (1).

"C'est une question de l'autonomie. C'est-à-dire la plus grande adaptation ça a été de ne pas accélérer à fond tout de suite, enfin tout le temps et puis d'anticiper tous les freinages pour avoir un freinage progressif et voilà ne pas faire des décharges trop rapides, pour avoir l'autonomie la plus longue possible quoi. Ça demande une conduite particulière. » (S2).

51 Tous les sujets n'utilisent pas systématiquement l'éco-conduite. Sa mise en œuvre peut dépendre des caractéristiques de la situation (autonomie restante, distance à parcourir) et de la manière dont l'individu les appréhende. Ces nouvelles pratiques et leur signification sont de plus en adéquation avec les valeurs des individus. En effet, l'écoconduite en plus de maximiser l'autonomie participe à la réduction de la pollution liée au déplacement, à travers l'optimisation de la consommation.

Pour des conducteurs (5), nous pouvons constater que ces nouveaux schèmes d'usage et d'action instrumentée développés avec la conduite de la VE sont favorablement reproduits avec leur voiture thermique.

« Pour la conduite écologique, vous savez, ne pas enfoncer le champignon, d'y aller progressivement, de rétrograder enfin utiliser le frein moteur, des choses comme ça, c'est des choses qu'on fait forcément plus avec une voiture électrique, parce qu'on augmente l'autonomie et donc on applique aussi avec l'autre véhicule 
maintenant. Enfin je n'ai jamais été un cow-boy en voiture non plus, mais je le suis encore beaucoup moins maintenant. » (S4)

53 À travers les stratégies mises en place par les conducteurs de VE pour pallier l'incertitude générée par l'autonomie limitée, nous pouvons observer un phénomène d'appropriation propice à son acceptation. Ainsi, l'activité se transforme à travers les nouveaux usages qui vont permettre aux sujets de contrôler ou de contourner des limites de la technologie. Ils développent des usages pour maitriser leur autonomie, leur consommation énergétique, anticiper les trajets qu'ils peuvent ou non réaliser. Cela passe par le développement de compétences spécifiques à l'instar de l'éco-conduite, et permet une valeur ajoutée à la conduite de la VE. Elle devient une activité mobilisant plus de savoir et savoir-faire (p. ex. éco-conduite, planification, connaissance approfondie des trajets) afin de contourner les limites la technologie et de maintenir, voire de développer des capacités d'action.

\subsubsection{Dangerosité perçue de l'absence de bruit comme source de genèse instrumentale}

54 L'absence de bruit de la VE est perçue de manière ambivalente par l'ensemble des sujets. Elle peut être une source de confort à la conduite ou potentiellement une source de danger. En effet, les conducteurs pensent que les usagers de la route non motorisés (piétons, cyclistes, etc.) ne vont pas détecter leur présence engendrant un risque d'accident. Selon (Cahour, 2010) les émotions influencent la créativité et le raisonnement, et pourraient jouer un rôle dans le développement de nouveaux usages. À titre d'exemple, l'inquiétude selon son intensité est susceptible d'avoir deux effets opposés selon les sujets : elle peut entrainer soit un comportement de prudence (Stephens \& Groegr, 2009), soit l'adoption d'une conduite plus risquée (Sahar, 2009). Dans le cas de la VE, nous avons pu observer l'émergence d'un comportement de vigilance accrue face à la perception du risque. Il est également adopté par les conducteurs spécifiquement avec la VE en réponse à une situation nouvelle, empreint d'inattendu, créée par l'absence de bruit moteur. On observe ici la formation d'une stratégie de coping en réponse à un inconfort émotionnel.

«Je vais quand même faire plus attention avec la voiture électrique. Enfin, on essaye bien toujours de faire attention aux piétons, mais là, il faut quand même faire gaffe parce qu'il y a le risque. Le bruit du moteur thermique nous offre une forme de sécurité, si le piéton ne nous voit pas, il va nous entendre. Là, on ne l'a plus. » (S9)

Elle peut au départ mobiliser des ressources attentionnelles importantes, mais est finalement intégrée par le sujet pour devenir un comportement normal, qu'ils reproduisent avec leur voiture thermique ( 5 sujets). Nous ne constatons donc pas seulement la création de nouveaux usages, mais de nouvelles normes d'usage par exemple en termes de vigilance envers les usagers de la route non motorisés.

«Dès qu'il y a quelqu'un par principe je ralentis, quand je passe dans des petites rues. Dès qu'ils me tournent le dos, donc quand on va dans le même sens, par peur que si la personne traverse inopinément, ça m'est déjà arrivé, heureusement que j'allais à une vitesse correcte, pour pouvoir m'arrêter. Voilà, j'anticipe tout simplement.

Ier : Vous faites ça plus qu'avec un véhicule thermique?

S5: Je dirais que ça m'a appris à le faire. Donc maintenant je le reproduis évidemment, mais c'est vrai qu'avant je ne faisais pas forcément attention à ça, parce que de toute façon un véhicule thermique ça fait du bruit, donc on l'entend arriver. »(S5). 
Nous observons un phénomène d'instrumentation par l'usage de la voiture électrique en vue de son utilisation la plus pertinente possible. Il participe à la genèse instrumentale de la VE et donc à son appropriation (Nogry, et al., 2015 ; Rabardel, 1995). Nous allons maintenant resituer ces nouveaux usages dans leur contexte et voir comment ils impactent les systèmes d'activité des individus.

\subsection{Le système d'activité pour l'analyse des impacts sociodomestiques de la voiture électrique}

L'usage d'une VE peut venir modifier les pratiques individuelles comme nous avons pu le voir, mais également l'organisation sociodomestique. Le modèle du système d'activités offre la possibilité d'appréhender l'usager de VE dans le contexte social, organisationnel et historique de réalisation de son activité.

Figure 3 : Système d'activité des utilisateurs de voiture électrique. Figure 3: Activity system of electric car users

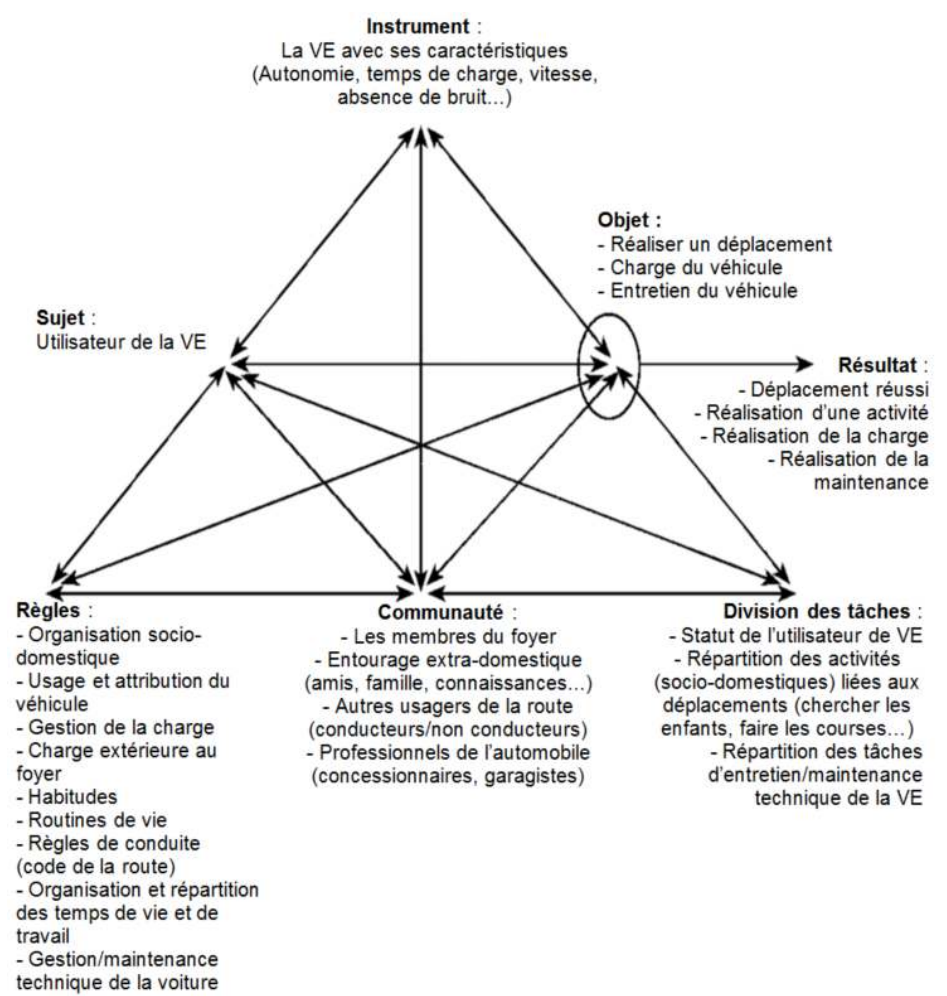

Avec la Figure 3, nous pouvons constater que le SA du conducteur est tout d'abord constitué d'un sujet, qui utilise une VE pour la réalisation de ces déplacements. Cette activité est inscrite dans une communauté, qui est composée la plupart du temps par les membres du foyer (SA sociodomestique). Selon l'activité réalisée, elle peut aussi être composée de l'entourage extra domestique, des usagers de la route, ou des professionnels de l'automobile. Ces différentes communautés sont régies par des règles qui peuvent impacter l'usage de la VE ou être impactées par celui-ci. Finalement, l'objet de l'activité, l'objectif du déplacement seront déterminés par les règles, mais également la division des tâches domestiques. 


\subsubsection{Stabilité et tensions au sein du système d'activité domestique}

59 Le but premier de l'usage d'un véhicule est la réalisation de déplacements. La situation des individus peut évoluer dans le temps et en même temps faire évoluer leurs besoins en déplacements, les usages du véhicule (Kaufmann, 1999), les activités qui leur sont associées et donc l'organisation domestique. Tous les sujets ont un usage très fréquent (quasi-journalier) de la VE.

Tableau 3 : Estimations par les conducteurs du nombre de km réalisés avec leur VE quotidiennement.

Table 3: Estimation by drivers of the number of $\mathrm{km}$ travelled daily with their electric car

\begin{tabular}{|l|c|c|c|c|c|c|c|c|c|c|}
\hline Sujet & S1 & S2 & S4 & S5 & S6 & S7 & S8 & S9 & S10 & Moyenne \\
\hline Estimation des Km journaliers & 32 & 24 & 70 & 20 & 80 & 40 & 50 & 30 & 80 & 47 \\
\hline Estimation de l'autonomie & 100 & 80 & 150 & 80 & 150 & 100 & 150 & 150 & 100 & 118 \\
\hline $\begin{array}{l}\text { Différence entre l'autonomie } \\
\text { et les Km journaliers }\end{array}$ & 68 & 56 & 80 & 60 & 70 & 60 & 100 & 120 & 20 & 71 \\
\hline
\end{tabular}

Les déplacements pendulaires (domicile/travail) sont en général les plus fréquents auxquels s'ajoutent en fonction de la situation de chaque individu; faire les courses, emmener ses enfants à l'école, rendre visite à des proches, etc. Nous pouvons constater avec le Tableau 3 une marge importante entre l'autonomie du véhicule déterminée par les sujets et celle dont ils ont besoin au quotidien. Elle permet une certaine souplesse et facilite les usages du véhicule au quotidien et l'organisation domestique.

«Par exemple, mon trajet qui fait 24 kilomètres, voilà, je regarde combien j'ai consommé, en général j'arrive chez moi, pour 24 kilomètres, j'ai consommé entre 25 et $30 \%$. [...] Voilà, entre 20 et $30 \%$, donc si j'ai consommé $20 \%$ la dernière fois, je sais que bon la batterie étant déchargée je vais consommer peut-être un peu plus sur le deuxième trajet, peut-être $35 \%$. J'aurais consommé $55 \%$ donc, avant d'atteindre le niveau bas à $20 \%$, il doit me rester $25 \%$ de batterie, donc je sais que je peux faire encore un petit trajet, donc aller à côté, à la boulangerie, ou voilà. » (S2)

61 À travers le discours des conducteurs, nous pouvons voir que l'usage de la VE s'intègre dans le quotidien des individus. En effet, tous ont déclaré l'utiliser pour tous les trajets qui rentrent dans leur périmètre d'autonomie. Son usage permet donc une continuité dans leurs pratiques quotidiennes. Cette intégration de la technologie dans les routines quotidiennes permet l'appropriation de cette technologie (Ling, 2004), et participe à sa domestication. Bien qui pouvait exister des tensions au début de l'usage, au moment où nous les avons interrogés, la VE n'a pas été source de tensions entre le sujet et l'objet de son activité. Elle a également permis le maintien de la division des tâches en permettant la réalisation des activités quotidiennes liées aux déplacements (p.ex. des courses alimentaires ou le transport des enfants).

62 Le système d'activité domestique reste relativement stable en lien avec la plupart des activités de déplacement médiatisées par la VE. Pourtant, certains types de déplacements, moins fréquents, peuvent engendrer des tensions, entre le pôle sujet et objet quand l'autonomie ne permet pas la réalisation du déplacement et donc de l'activité (Tableau 4). 
Tableau4 : Tensions au sein du SA domestique, liées à l'autonomie de la voiture électrique. Table 4: Tensions in domestic activity system, related to electric car's autonomy

\begin{tabular}{|l|l|l|}
\hline Schématisation & Niveau de contradiction & Description \\
\hline & $\begin{array}{l}\text { Secondaire : } \\
\text { Sujet / Instrument } \\
\text { Instrument / Objet } \\
\text { Sujet / Objet }\end{array}$ & $\begin{array}{l}\text { La technologie du fait de ses } \\
\text { caractéristiques en termes d'autonomie et } \\
\text { de temps de charge ne permet pas la } \\
\text { réalisation du déplacement }\end{array}$ \\
\hline
\end{tabular}

63 L'autonomie et le système de recharge peuvent rendre impossible un déplacement avec la voiture électrique pour différentes raisons. Son non-usage peut simplement reposer sur la contrainte spatiale (évoqué par 9 sujets). En d'autres termes, la nécessité de réaliser un déplacement, dont la distance, est supérieure à l'autonomie de la voiture (9). Les sujets évoquent par exemple des vacances ou sorties (5), ou encore des déplacements professionnels (2). Une autre raison peut être l'incertitude de la faisabilité du déplacement (5) quand le conducteur doit réaliser un déplacement imprévu (1) qui n'appartient pas aux déplacements qu'il connaît. Cela va engendrer de l'anxiété du fait de l'incertitude d'avoir suffisamment d'autonomie (5) pour réaliser le trajet. Enfin, les critères de non-usage lié à la charge (4) peuvent se décliner en oubli de charge (1) qui rend le véhicule indisponible, ou encore impossibilité de charge (3) au lieu de destination.

L'ensemble de notre échantillon possède en plus de la voiture électrique une voiture thermique. Face à la limitation de l'autonomie et aux tensions que cela peut faire naître au sein du SA domestique, les sujets changent donc de véhicule quand cela est possible (véhicule thermique disponible). Dans la plupart des cas, cela permet de maintenir le flux d'activités quotidiennes des individus liées aux déplacements. Plus qu'un simple recours, pour certains sujets (4), posséder une voiture thermique est un impératif. Le fait que tous les foyers soient multimotorisés réduit les risques de rejet de la VE, mais va engendrer des transformations au sein du pôle règles et division des tâches. La division des tâches va être adaptée en fonction des possibilités des différents véhicules (instrument), et des activités à accomplir (objet) pour adapter son usage à l'organisation sociodomestique et assurer le maintien de la réalisation des activités liées aux déplacements. Cela permet de maintenir l'équilibre au sein du SA. Mais ce changement de véhicules est susceptible d'engendrer d'autres tensions au sein de l'organisation domestique et entre les acteurs en lien avec l'attribution des véhicules.

\subsubsection{Redéfinition des règles d'attribution et de la division des tâches}

L'attribution de l'utilisation de la VE fait l'objet d'une organisation consensuelle au sein des foyers. Bien que des transformations dans le SA sociodomestique puissent apparaitre, nous pouvons constater une continuité dans les pratiques des individus. L'absence de tension au sein du SA domestique, est due aux transformations au sein des différents pôles intervenus principalement dans les premiers temps de l'usage. Elles ont été engendrées par les contraintes de l'instrument et concernent principalement le pôle règles avec l'attribution de la $\mathrm{VE}$, mais également le pôle divisions des tâches, avec par exemple une redéfinition du statut d'usager principal du véhicule. L'effet des contraintes de la VE sur l'organisation sociodomestique et le flux d'activités liées au déplacement est ainsi minimisé. Les règles d'attribution sont construites en fonction d'un arbitrage effectué par les individus entre la situation et un certain nombre de critères. Il existe une hiérarchisation des critères d'attribution, par exemple le statut de l'utilisateur 
(utilisateur principal) peut être plus important que les priorités des individus (confort d'usage), ou que les enjeux pour le foyer (optimisation de l'usage pour des raisons financières).

Au sein du foyer, un individu peut être désigné comme l'utilisateur principal (7) : il s'agit de la personne dont les déplacements pendulaires sont les plus compatibles avec la VE (p.ex. autonomie, confort). Pour cet utilisateur, la réalisation de déplacements imprévus nécessite alors une coordination avec un autre membre du foyer, ce qui peut potentiellement être source de tensions intra-SA.

67 L'attribution de la VE peut également dépendre de déterminants liés à l'usage (5). Ce peut donc être le type de déplacement (4) qui va déterminer l'attribution de la VE (court trajet, peu de passagers). Des foyers vont chercher à maximiser l'usage de la VE (3) qui sera utilisé prioritairement par rapport à leur voiture thermique pour des raisons de coût d'utilisation. Le véhicule va donc être utilisé pour le plus de déplacements possible. Des conditions facilitatrices liées aux lieux de destination peuvent également jouer un rôle dans l'attribution du véhicule. Des sujets évoquent le fait d'avoir une place de parking avantageuse pour la VE (1), ou encore une possibilité de charge gratuite (1).

"Je peux la charger à mon travail et ce n'est pas le cas de ma femme. Donc c'est déjà une raison importante. Et pour le trajet domicile travail c'est la raison principale. Ensuite en dehors de ça, ma femme l'utilise comme moi. Si elle a un trajet à faire et que la VE est disponible, elle la prend. » (S8)

Les caractéristiques de la VE (3) peuvent également déterminer son attribution (p. ex. performances, utilisabilité). Ainsi, elle peut être utilisée par l'individu ayant le plus d'expérience ou de compétence pour son usage. Mais dans d'autres cas, il peut s'agir de la personne la plus encline à l'abandon des éléments de confort pour l'optimisation de l'autonomie.

«Mon épouse une fois elle l'avait, l'hiver surtout, quand on met du chauffage, pour désembuer tout ça, on ne peut pas le mettre constamment, parce qu'on réduit d'un tiers l'autonomie, donc on est obligés de, on le met, on l'enlève, dès que ça revient, on le remet, on enlève le chauffage, on ne le laisse, pas, enfin on n'en met pas beaucoup.» (S7)

"Mon épouse une fois elle l'avait, l'hiver surtout, quand on met du chauffage, pour désembuer tout ça, on ne peut pas le mettre constamment, parce qu'on réduit d'un tiers l'autonomie, donc on est obligés de, on le met, on l'enlève, dès que ça revient, on le remet, on enlève le chauffage, on ne le laisse, pas, enfin on n'en met pas beaucoup.» (S7)

69 À l'inverse ces caractéristiques peuvent conduire à une «non-attribution » de la VE (2) à certains membres du foyer. À titre d'exemple, un individu ne veut pas que ses enfants en cours d'apprentissage de la conduite l'utilisent, car elle ne permet pas d'acquérir certaines compétences nécessaires avec les voitures thermiques. Ces deux types de véhicules diffèrent en effet dans la mobilisation de certains schèmes d'usage.

"J'ai deux enfants qui sont en train de passer le permis là et du coup, ils ne peuvent pas conduire le véhicule électrique, parce que ce n'est pas un bon entraînement pour passer le permis sur une boite manuelle » (S6).

70 Les règles d'attribution de la VE peuvent être source de tensions au sein du SA domestique (Tableau 5). À titre d'exemple, quand deux membres du foyer ont besoin de la voiture thermique pour un long déplacement au même moment, cela peut créer des tensions. Elles se situent entre le sujet, la communauté (membres du foyer) et les règles 
(attributions des véhicules). Ces tensions peuvent également engendrer des modifications dans la réalisation de certaines activités (pôle objet).

Tableau 5 : Tensions au sein du SA liées à l'attribution des véhicules.

Table 5: Tensions in activity system related to the allocation of vehicles

\begin{tabular}{|c|c|c|}
\hline Schématisation & Niveau de contradiction & Description \\
\hline & $\begin{array}{l}\text { Secondaire : } \\
\text { Sujet / Communauté } \\
\text { Sujet / Règles } \\
\text { Communauté / Règles } \\
\text { Sujet / Objet }\end{array}$ & $\begin{array}{l}\text { Le sujet et un membre du foyer ont besoin } \\
\text { d'une VT pour un déplacement impossible } \\
\text { avec la VE en même temps. } \\
\text { Cela va créer des tensions entre le sujet et le } \\
\text { membre du foyer, dont les enjeux seront les } \\
\text { règles d'attribution du véhicule. }\end{array}$ \\
\hline
\end{tabular}

Bien qu'il existe des règles, cela ne fige pas l'attribution des véhicules. La multimotorisation des foyers facilite une certaine souplesse dans l'organisation de l'attribution des véhicules qui va pouvoir évoluer en fonction de la situation (3). Ainsi, les voitures peuvent être attribuées en fonction des besoins en autonomie (2) ou encore être utilisées quand celle-ci est disponible, que le conducteur "principal» n'en a pas l'utilité (2). Cela permet le dépassement des tensions au sein du SA et le maintien du flux d'activités domestiques, participant ainsi à une acceptation positive de la VE d'un point de vue social.

\subsubsection{Suppression de temps contraint}

La charge de la VE peut avoir des effets sur l'organisation domestique et les pratiques quotidiennes en lien avec les déplacements. En effet, celle-ci requiert l'accès à une prise électrique et une immobilisation du véhicule qui peut varier d'une trentaine de minutes à plus d'une dizaine d'heures. La non-charge des batteries peut mettre à mal le flux d'activités liées aux déplacements, sa remédiation nécessite l'usage d'un autre mode de transport tel que la voiture thermique. Des tensions peuvent alors apparaitre dans la division des tâches et au sein du collectif à travers les règles d'attribution des véhicules. Pour pallier cela, des stratégies de charge sont mises en place par les individus. Certains chargent systématiquement leur véhicule, alors que d'autres le chargent en fonction du niveau de l'autonomie.

Le moment de charge privilégié par la quasi-totalité des sujets (8) est la nuit. Ce choix incombe à un aspect monétaire (le prix de l'électricité est réduit), ainsi qu'à l'absence de déplacements pendant cette période. Cette organisation ne contraint pas les individus qui branchent leur véhicule quand ils rentrent à leur domicile et gèrent la charge à l'aide d'un "programmateur». Mais cette période de charge n'est pas statique, elle peut dépendre des besoins des individus, et peut donc être amenée à évoluer.

« Au domicile, le soir quand je rentre, j'ai programmé ça charge de nuit, parce que moi j'ai un tarif entre minuit et 5 heures qui est réduit. Donc elle se charge automatiquement entre minuit et 6 heures du matin » (S10).

74 La charge qui généralement est réalisée au domicile des sujets est perçue comme une opportunité. Dans leurs usages quotidiens, les utilisateurs la comparent aux pratiques de charge d'autres appareils électriques tels que les téléphones portables.

«C'est un peu comme les téléphones portables maintenant quoi. Le téléphone portable maintenant, on le branche tous les soirs. La voiture on la branche le soir et c'est terminé, on ne passe plus à la pompe. »(S9). 
Les stratégies de charge relèvent en premier lieu de la nécessité d'avoir un véhicule opérationnel pour la réalisation de déplacement et en second lieu, d'un motif monétaire visant à optimiser le coût à l'usage. Elles s'intègrent dans le quotidien des individus, deviennent des routines et ne créent pas de concurrences intra-pratiques. Au contraire, elle transforme du temps contraint en temps libre en supprimant le déplacement pour le réapprovisionnement comme pour les voitures thermiques. Nguyen (2013) montre que le rapport aux stations-service peut engendrer un inconfort émotionnel, car vécu comme désagréable (odeur, toucher). Il peut engendrer du stress en lien avec un sentiment de pression temporelle (attente d'autrui). Le système de charge de la VE permet de supprimer une activité (réapprovisionnement d'une voiture thermique) et en crée une autre jugée moins contraignante. La charge à domicile jouerait donc un rôle positif dans le maintien de l'usage de la VE et donc de son acceptation. En revanche, la charge à l'extérieur du domicile peut ajouter des sources de tensions comme nous allons le détailler.

\subsection{Les effets sociaux, identitaires et statutaires de l'usage de la voiture électrique à partir de l'analyse des SA extra-domestiques}

76 L'usage de la VE se déploie dans différents contextes, pouvant être extérieurs au foyer. Ces autres SA ont leurs propres règles, acteurs, statuts pouvant être impactés par ce type de véhicule. Il en résulte une modification de l'image que renvoie le sujet et de son identité de conducteur.

\subsection{1. Élaboration de règles autour de la charge du véhicule en dehors du domicile}

La charge de la VE a des effets sur les pratiques quotidiennes des individus et l'organisation sociodomestique. Les stratégies visant son optimisation ont pour but de garantir la réalisation des activités liées aux déplacements. Cette optimisation peut passer par la réalisation de charges ailleurs qu'au domicile.

Tableau 6 : Lieux de charge utilisés par les sujets. Table 6: Charging locations used by subjects

\begin{tabular}{|l|c|c|c|c|c|c|c|c|c|c|}
\hline Lieux de charge & S1 & S2 & S4 & S5 & S6 & S7 & S8 & S9 & S10 & total \\
\hline Au domicile & & & & & & & & & & $\mathbf{9}$ \\
\hline Chez des amis & & & & & & & & & & $\mathbf{5}$ \\
\hline Dans la famille & & & & & & & & & & $\mathbf{5}$ \\
\hline Sur le lieu de travail & & & & & & & & & & $\mathbf{4}$ \\
\hline Sur des bornes publiques & & & & & & & & & & $\mathbf{4}$ \\
\hline
\end{tabular}
Cela peut être dû au manque d'opportunité ou l'absence de nécessité, la plupart des déplacements étant réalisés dans le périmètre d'autonomie du véhicule. L'impossibilité d'accès à des points de charge extérieurs ou leur défaillance est également évoquée par des sujets ainsi que l'inconfort émotionnel que peut créer la charge chez autrui (clients, collègues, supérieur, mais également amis, famille, etc.).

Le non-recours à la charge au domicile d'autrui est généralement dû à la gêne occasionnée par la demande (4). 
"Je peux faire mes déplacements professionnels, mais quand on vous appelle, "tu peux passer?", bah je ne peux pas parce que je n'ai pas assez d'autonomie. Vous pouvez quand même la brancher chez n'importe quel privé si vous voulez, mais bon je ne vais pas chez des personnes et je leur demande si je peux brancher ma voiture, c'est un peu délicat. À part si c'est des amis. » (S10)

80

Selon les individus, la gêne ressentie peut être différente en fonction du rapport qu'ils ont avec la personne. Ce type de charge peut être perçu par les sujets comme une transgression de règles implicites, historiquement construites, concernant le réapprovisionnement de son véhicule.

«C'est quand même un peu gênant, je n'ose pas leur dire on va se brancher pour recharger. Il y a ce rapport encore, on n'arrive pas en demandant un bidon de gasoil chez quelqu'un. Sauf si on a un pépin quoi, là on donne volontiers. On ne va pas rester... »(S1).

81

La charge de la VE au domicile d'autrui peut engendrer des tensions au sein du SA composé par le sujet et l'individu chez qui est réalisé la charge. Elles émergent entre le pôle sujet, la communauté (amis, famille, connaissance... chez qui la charge pourrait être réalisée), l'objet de l'activité (la charge) et les règles (Tableau 7).

Tableau 7 : Tensions au sein du SA liées à la charge chez autrui.

Table 7: Tensions in activity system related to the charge at other people's home

\begin{tabular}{|c|c|c|}
\hline Schématisation & Niveau de contradiction & Description \\
\hline & $\begin{array}{l}\text { Secondaire : } \\
\text { Sujet / Communauté } \\
\text { Sujet / Règles } \\
\text { Communauté / Règles } \\
\text { Sujet / Objet }\end{array}$ & $\begin{array}{l}\text { Le sujet désire charger son véhicule au domicile de la } \\
\text { personne chez qui il se rend, pour conserver un } \\
\text { niveau d'autonomie optimal, ou par nécessité pour } \\
\text { réaliser le trajet retour. } \\
\text { Cette charge chez autrui peut être considérée par le } \\
\text { sujet et/ou la personne visitée comme inconvenante, } \\
\text { en contradiction avec les règles régissant leurs } \\
\text { rapports. }\end{array}$ \\
\hline
\end{tabular}

Un sujet n'a pas pu remédier à ces tensions principalement à cause de la gêne occasionnée par la demande. La charge chez autrui pour ce sujet une transgression de règles implicite. Cela peut conduire à l'abandon de la pratique de la charge extérieure et dans certains cas au non-usage de la VE pour certains déplacements. Au contraire, pour d'autres sujets, ou pour d'autres systèmes d'activités, nous pouvons voir que ces tensions ont pu amener des transformations du système, redéfinissant les règles et normes établies au sein desquelles la charge chez autrui devient acceptable. La création de règles implicites ou explicites peut différer selon les personnes, leur environnement social, ou les situations. Il n'y a pas de normes pour ce type de charge, mais des règles construites de manière ponctuelle.

\subsubsection{Nouveaux rapports aux usagers de la route pour de nouveaux usages}

83 L'éco-conduite que les conducteurs ont développée peut engendrer une modification du rapport avec les autres conducteurs de véhicules motorisés (notamment à cause des accélérations ou de la vitesse modérée). Des tensions entre différents pôles au sein de ce SA émergent entre la communauté composée des usagers de la route, les règles implicites et explicites qui régissent leurs interactions et le sujet (Tableau 8). 
Tableau 8 : Tensions au sein du SA liées aux autres usagers de la route. Table 8: Tensions in activity system related to other road users

\begin{tabular}{|l|l|l|}
\hline Schématisation & Niveau de contradiction & Description \\
\hline & $\begin{array}{l}\text { À travers les stratégies mises en place par les } \\
\text { conducteur (vigilance accrue, éco-conduite) en } \\
\text { réponse aux caractéristiques de la VE (absence de } \\
\text { bruit ou autonomie), les sujets peuvent déroger aux } \\
\text { règles régissant les rapports entre les usagers de la } \\
\text { route, engendrant ainsi des tensions entre ces } \\
\text { différents pôles. }\end{array}$ \\
$\begin{array}{l}\text { Sujet / Communauté } \\
\text { Communauté / Règles }\end{array}$ &
\end{tabular}
vont préférer modifier leur activité (p.ex. modification d'itinéraire). Ainsi ils évitent les situations ou le style de conduite " éco " va avoir le plus d'impact sur les autres usagers (principalement les autoroutes). Les possesseurs de VE vont donc faire évoluer leur activité de conduite pour qu'elle ne rentre pas en conflit avec les règles implicites qui régissent les rapports entre usagers de la route motorisés.

« Il y a des voitures, comme je ne roule pas très vite pour préserver les batteries on va dire, rester dans le vert dans l'économètre, c'est vrai que les autres véhicules se demandent qu'est-ce que c'est ce papy qui se traîne sur la route. Donc c'est ça quoi, les gens sont plus énervés, parce que je ne suis pas en train de rouler à 90 , juste à la limite du flash quoi. » (S2).

Le rapport aux usagers de la route non-motorisés (piétons, cyclistes, etc.) peut également se voir affecté dans certaines circonstances. Contrairement au rapport avec les usagers de la route motorisé, nous pouvons constater une ambivalence dans les interactions. La transgression des règles formelles du Code de la route par des piétons (p.ex. marcher sur les trottoirs ${ }^{5}$, traverser la route selon des règles précises ${ }^{6}$ ) peut dégrader les relations avec les conducteurs. La dangerosité perçue de ces comportements est exacerbée par les caractéristiques de la VE à savoir l'absence de bruit et l'incertitude d'être détecté, ce qui engendre un inconfort émotionnel chez les conducteurs.

Pour remédier à ces situations, certains conducteurs disposent dans leur véhicule d'avertisseurs sonores prévus à cet effet: 2 sujets utilisent un bruit artificiel à faible vitesse (moins de $30 \mathrm{~km} / \mathrm{h}$ ), 1 sujet utilise une alarme de recul lors de la réalisation de manœuvres, et 1 sujet utilise un «klaxon pour piétons». Les sujets n'ayant pas d'équipements spécifiques dans leur véhicule utilisent d'autres stratégies. Ainsi, 4 sujets utilisent leur klaxon pour signaler leur présence. L'utilisation de cet avertisseur peut être perçue comme agressive par les piétons, il y a donc une restriction de son usage. Finalement un sujet a mis en place une stratégie pour contourner ce problème qui consiste à mettre la radio de son véhicule suffisamment fort pour être entendu. Cela témoigne d'un phénomène de "catachrèse " (Rabardel, 1995), où l'usage prévu lors à la conception d'un artéfact est détourné pour un autre usage. Les sujets ont donc créé de nouvelles modalités d'interactions et de cohabitations avec les usagers de la route non motorisés.

«Il y a des gens qui marchent sur la route et moi j'arrive derrière et ils ne me voient pas. Donc je suis obligé de klaxonner pour qu'ils partent sur le trottoir, pour qu'ils me laissent la place. Ils me disent qu'ils ne m'ont pas entendu, enfin ils me le signifient avec des gestes qu'ils ne m'ont pas entendus. » (S5).

Les rapports aux usagers de la route ne sont pas tous à l'origine d'éprouvés négatifs. $\mathrm{Au}$ contraire les conducteurs attirent la curiosité d'autrui engendrant de nouvelles interactions, le véhicule est alors vecteur de rapports sociaux. 
"Ça nous est déjà arrivé à des feux rouges ou des choses comme ça, il y a des gens qui se retournent, qui posent la question ou qui font des gestes pour dire que ça va bien, oui ça arrive. » (S4).

88 Au final, la VE à travers ses caractéristiques (autonomie, propriétés acoustiques...), mais également les stratégies et les nouveaux usages qui sont développés (éco-conduite, vigilance accrue...), va transformer les rapports qu'ont les conducteurs avec les autres usagers de la route. Ainsi, des tensions peuvent émergées au sein du SA qu'ils constituent. Cela peut engendrer une dégradation des rapports interindividuels ou encore un inconfort émotionnel chez le conducteur. Ce dernier va développer des stratégies de remédiation qui peuvent être une évolution de l'activité ou la modification des usages. Cela peut passer par ne plus utiliser certains itinéraires, ou encore, l'utilisation de la radio comme indicateur de présence du véhicule. Ces changements opérés par le sujet vont lui permettre de se maintenir au sein de ce système en supprimant les zones de tensions évitant ainsi le rejet de la technologie.

\subsubsection{Redéfinitions des rôles et du statut des acteurs de la voiture électrique}

Les VE, comme les voitures thermiques, nécessitent la réalisation d'un certain nombre de tâches pour garantir leur bon fonctionnement. Celles-ci impliquent l'intervention d'acteurs, de professionnels spécialisés dans ce domaine. L'utilisation d'une VE va engendrer des transformations dans les rapports des conducteurs avec d'autres acteurs avec une modification des statuts des individus, mais aussi le développement de nouvelles compétences.

90 De nombreux sujets (7) évoquent le peu d'entretien que nécessitent les VE à la différence des voitures thermiques, et de ce fait ils les perçoivent comme plus fiables. Cela permet la suppression de contraintes, notamment liées à l'immobilisation du véhicule pour son entretien (qui paraît également moins coûteux). L'organisation domestique se trouve ainsi facilitée ce qui favorise le maintien de l'usage du véhicule. Nous avons donc ici un nouvel exemple de transformation de temps contraint en temps libre avec l'introduction de la VE dans le foyer et les pratiques qui lui sont associées.

« Il n'y a quasiment pas d'entretien sur ces véhicules. Après, on n'a pas beaucoup de recul non plus. Mais donc au bout de l'année, je l'ai amené effectivement pour la révision des 1 an. Sachant que contrairement à un moteur thermique, il y a beaucoup moins d'entretien sur la motorisation, du coup, l'entretien, la visite a été vachement pas onéreuse du tout. Et ils m'ont expliqué qu'a priori, à part les freins, les pneus, enfin ce qu'on utilise régulièrement sur un véhicule, il n'y a pas tout le système de filtres, de courroie, etc., qu'on trouve sur les véhicules classiques, qui sont chers à entretenir. A priori, c'est un moindre entretien. »(S6).

91 Cet avantage est cependant pondéré par la perception des acteurs professionnels de l'automobile (garagistes, concessionnaires) comme insuffisamment experts dans la technologie. Si l'on considère le SA formé par les sujets et ces professionnels de l'automobile, nous pouvons constater une tension entre les pôles sujets, communauté (professionnels de l'automobile), les règles (gestion/maintenance) et la division des tâches (maintenance) (Tableau 9). 
Tableau 9 : Tensions au sein du SA liées à l'attribution du statut de spécialiste. Table 9: Tensions in activity system related to the position as electric car expert

\begin{tabular}{|c|c|c|}
\hline Schématisation & Niveau de contradiction & Description \\
\hline & $\begin{array}{l}\text { Secondaire : } \\
\text { Sujet / Communauté } \\
\text { Sujet / Règles } \\
\text { Communauté / Règles } \\
\text { Sujet / Objet }\end{array}$ & $\begin{array}{l}\text { Les professionnels de l'automobile ne } \\
\text { pouvant pas répondre aux attentes des sujets } \\
\text { en termes de renseignement et de } \\
\text { gestion/maintenance du véhicule, il va y } \\
\text { avoir une remise en cause de leur statut de } \\
\text { spécialiste qui est parfois réattribué aux } \\
\text { sujets }\end{array}$ \\
\hline
\end{tabular}

La perception du manque d'expertise engendre chez les sujets un manque de confiance et une remise en question du statut de spécialiste et donc de son identité. Cela étant due par exemple à leur incapacité à pouvoir répondre aux interrogations des sujets, mais également à l'écart entre l'autonomie annoncée et celle déterminée par les conducteurs. Ainsi, des difficultés à trouver une personne compétente sont évoquées par un sujet. D'autres (4) déclarent ne pas avoir eu de difficulté, mais font part de leur impossibilité de " choisir leur garagiste » (3) au vu du faible nombre de professionnels compétents.

«Des fois, on a l'impression qu'ils n'en savent pas plus que nous. Peut-être que je généralise, ça va venir, parce que, comme ils avaient qu'une voiture ou deux par garage, c'est vrai que, c'est comme un chirurgien qui opère que deux fois par an telle intervention, ce n'est pas le meilleur. Je ne dis pas qu'il va rater son truc, mais voilà, il est moins à même de régler si d'un coup il se met à y avoir un problème au cours de l'intervention quoi, il a moins d'expérience. » (S1)

93 D'autres transformations peuvent survenir au sein du SA. Pour un type d'utilisateurs de $\mathrm{VE}$, nous observons une évolution au niveau de la division des tâches où le rôle du professionnel qui est de réalisé entretien du véhicule est attribué à l'utilisateur. Les possesseurs de VE d'ancienne génération (2) réalisent la quasi-totalité des opérations d'entretien sur leur véhicule. Ils possèdent des connaissances approfondies relatives aux batteries, à la charge, au groupe motopropulseur et aux opérations de maintenance. Cela tend à montrer une évolution des connaissances et compétences des individus, permettant une forme d'autonomie dans la gestion du véhicule.

\subsubsection{La construction d'une identité positive}

94 Les caractéristiques de la VE engendrent de nouvelles pratiques, elles-mêmes à la source de transformations au sein de systèmes d'activités dans lesquels elles sont impliquées. Ces transformations peuvent venir modifier l'identité de l'utilisateur à travers la signification de ses usages (Orlikowski, 1991), participant ainsi davantage à l'appropriation de la technologie. La VE n'est alors plus pour le sujet un simple instrument lui permettant de réaliser son activité de déplacement, elle lui donne de nouvelles significations, notamment en termes de préservation de l'environnement. Cet aspect étant en accord avec les valeurs des sujets il y a donc une revalorisation de l'activité de conduite et de l'identité du conducteur. Cela passe par le regard d'autrui dans lequel le conducteur de voiture électrique devient un utilisateur d'une technologie spécifique et proenvironnementale contribuant à la résolution de problèmes environnementaux. L'adéquation de la technologie avec l'individu en termes d'identité d'usage, de performances, mais également de signification des usages (préservation de l'environnement), renforce l'appropriation de la technologie et le maintien de son utilisation. 

autrui.

« Nous on prône complètement la VE. Nous on la vend, mais on peut servir de commerciaux. Nous au contraire, on les aide à réfléchir un peu. » (S1).

Cette promotion passe en premier lieu par la transmission d'informations à des nonusagers. À travers le discours des sujets, nous pouvons voir le décalage existant entre les perceptions d'autrui et celles des conducteurs. Leur discours nous renseigne également sur la manière dont est vécu ce décalage par les utilisateurs.

«Je me dis juste que parfois on fait des faux procès des véhicules électriques. [...] Aujourd'hui, les gens disent que 100 kilomètres d'autonomie, ce n'est pas suffisant, voilà, je ne peux pas m'en servir. Faux, je leur pose la question, combien de jours par an, vous faites plus de 100 kilomètres par jour? Et en gros, si on réfléchit bien, c'est très peu de jours. » (S8).

D'une manière générale, les conducteurs de VE font face aux méconnaissances des nonusagers. Leur usage leur donne à la fois un statut de précurseur, mais également de spécialiste, reconnu par autrui. Ils vont ainsi faire part de leurs connaissances et expériences d'usage afin de dissiper des idées reçues ou satisfaire des interrogations. Cette démarche est réalisée de manière positive par les utilisateurs. Elle témoigne de la création d'activités d'information/promotion de la VE qui associent les usages de la technologie, les compétences et connaissances qu'ils en ont développées et la signification de ces nouvelles pratiques. L'association de ces éléments témoigne de l'appropriation des usages de la technologie.

«J'aime ça. Parce qu'en fait on me pose beaucoup de questions encore aujourd'hui.

On m'en pose vraiment beaucoup, j'aime ça et c'est vrai qu'à chaque fois, j'ai

l'impression de convaincre les gens du fait que ce soit pratique. J'ai toujours la réponse »(S5).

Ces effets sociaux et identitaires avec la modification du statut social des conducteurs de VE nous amènent à déduire qu'ils auraient atteint la phase de conservation (Ling, 2004). Cette dernière phase du processus de domestication nous permet de convenir de l'intégration de la VE dans le quotidien des individus et donc de sa domestication (Silverstone, \& Haddon, 1996). Cette identité positive de conducteur de la VE est favorable à son acceptation.

\section{Discussion et conclusion}

Nous avons cherché à appréhender le maintien de l'usage de la VE dans une temporalité relativement longue. L'étude de l'acceptation ne se limite pas à l'étude de l'objet technique, mais aux activités qui sont impactées par son usage, qu'elles soient anciennes, nouvelles, transformées ou empêchées. Cela a été rendu possible par l'application de l'approche des systèmes d'activité (Engeström, 1987) à un contexte d'usage sociodomestique. Les VE, même si elles sont proches des voitures thermiques s'en différencient en de nombreux points que ce soit au niveau de la conduite, du réapprovisionnement en énergie, ou de la manière d'appréhender les déplacements.

Notre méthodologie est basée sur la combinaison d'un premier entretien général suivi d'un second approfondi à l'aide de techniques inspirées par les entretiens d'explicitation et les incidents critiques. Elle nous a permis d'appréhender en détail les évolutions des activités liées aux déplacements ainsi que leurs effets sur l'acceptation de la VE. Afin 
d'avoir un regard plus global sur ces transformations, mais également l'effet qu'elles peuvent avoir pour les individus, pour les groupes, leurs interactions et la manière dont les individus vont se positionner dans cette nouvelle configuration, nous nous sommes appuyés sur l'approche de l'acceptation située (Bobillier-Chaumon, 2016). Elle est ancrée dans le réel de l'activité et définit quatre grandes dimensions à prendre en compte pour comprendre le rejet ou le maintien de l'usage (individuelle, organisationnelle, relationnelle et identitaire). Nous avons identifié dans les discours des redondances qui nous permettent de créer un faisceau d'indices nous renseignant sur la tendance dominante de l'acceptation située de la VE.

Dimension individuelle : L'autonomie de la VE ainsi que sa charge peuvent contraindre l'activité. Pour pallier à cela et réduire l'incertitude pouvant être générée, les conducteurs ont développé différentes stratégies de gestion de l'autonomie, ainsi que de gestion de la charge. Ces stratégies créent de nouvelles activités de planification et de gestion des déplacements, ainsi que de nouveaux schèmes d'usage. Il en résulte une facilitation de la réalisation des tâches de contrôles du véhicule lors de la conduite (Allen, Lunenfeld, \& Alexander, 1971). Cette facilitation permet de réinvestir des ressources cognitives dans la vigilance accrue envers les usagers de la route non motorisés, réduisant encore la charge émotionnelle. Avec l'utilisation d'une VE, l'activité de déplacement ne se dégrade pas, elle se transforme, s'enrichit, mais reste favorable à l'individu, ce qui participerait au maintien de son usage. Des éléments pouvant produire un inconfort émotionnel en lien notamment avec l'incertitude (autonomie, consommation du véhicule, détection par les piétons/cyclistes) ont été dépassés par les sujets avec la mise en place de stratégies de coping.

Dimension organisationnelle : La VE est le véhicule le plus fréquemment utilisé par l'ensemble des sujets, et il leur permet de réaliser la majorité de leurs déplacements. Cela est facilité par les possibilités de réapprovisionnement en énergie (charge à domicile, charge extérieure), mais également grâce à une certaine souplesse dans l'organisation domestique. La VE permet à la plupart des individus suffisamment d'autonomie et de marge de manœuvre pour réaliser leurs activités, dans ce sens, elle est une ressource. Les possibilités offertes par la VE, ainsi que l'organisation sociodomestique mise en place favorise son adaptation aux situations. Cela passe également par la possession et l'utilisation d'un second véhicule. Ainsi nous pouvons nous interroger si le maintien de l'organisation reste possible avec seulement l'usage d'une VE. Cette dernière peut être intégrée dans les activités quotidiennes des individus nécessitant parfois des ajustements. L'organisation sociodomestique des possesseurs de VE n'est donc pas mise à mal, ce qui favoriserait le maintien de l'usage de ce type de véhicule.

Dimension relationnelle : Les participants témoignent d'une méconnaissance de la VE par de nombreuses personnes, pour ces derniers elle est source d'étonnement et d'interrogations. Avec l'expérience de cette technologie, les utilisateurs deviennent des spécialistes et de manière volontaire ou non ses "promoteurs ». Dans cette perspective, ce type de véhicule est une source d'interactions sociales, sous forme d'informations fournies à autrui que ce soit des proches ou des inconnus dans la rue. Par ailleurs, les nouveaux comportements d'usage de la VE vont modifier les relations avec d'autres usagers de la route. Elles peuvent se dégrader dans certaines conditions (p.ex. autoroute) où l'éco-conduite peut remettre en cause des règles implicites (ne pas ralentir le flux de circulation). Nous constatons donc une ambivalence dans les interactions qui sont créées ou impactées par la VE, avec des individus extérieurs au foyer. Ces interactions restant 
cependant marginales, elles semblent avoir peu d'effet dans le maintien de l'usage de la VE. Les relations domestiques sont quant à elles peu impactées par l'usage de ce type de véhicule. Aucune concurrence intra-pratique (Shove, Pantzar, \& Watson, 2012) n'émerge grâce par exemple à une répartition consensuelle de l'usage des véhicules ce qui facilite le maintien de son usage.

Dimension identitaire : L'identité du conducteur va se trouver changée à travers la modification de la signification des usages (Orlikowski, 1991). Les sujets se reconnaissent dans leurs usages qui correspondent à leurs valeurs environnementales, mais également à leur conception de la conduite (" reposante », « douce», " confortable », etc.). L'usage de cette technologie véhicule d'autres significations pour des sujets en termes de promotion de la nouveauté, et de mode de transport alternatif. L'identité des conducteurs de VE est également construite à partir de la reconnaissance d'autrui. Ils sont perçus comme spécialistes de la technologie, parfois plus reconnus que certains professionnels. Des individus proches ou non des sujets reconnaissent leurs expertises et émettent des demandes d'informations, de partage de leurs connaissances et expériences. La reconnaissance des conducteurs de VE passe également par l'aspect pro-environnemental attaché au véhicule. L'identité du sujet est donc favorablement impactée par l'utilisation d'une VE, participant ainsi au maintien de son usage. Avec leur usage d'une technologie perçue comme pro-environnementale, les sujets vont participer à la promotion de la réduction de la pollution et de l'usage d'autres types de véhicule de manière passive. Cette participation est également active à travers leurs discours d'information et de promotion de la technologie.

En conclusion, il ressort de nos résultats que les caractéristiques, les opportunités, mais surtout les contraintes et l'usage de la VE vont avoir de nombreux effets sur les individus et la réalisation de leurs activités. En effet, c'est en voulant dépasser ces contraintes que les individus vont développer de nouvelles connaissances, et compétences qui vont s'inclure dans leurs usages et même se transmettre à d'autres. Les spécificités de la VE en termes d'autonomie et de charge ainsi que les opportunités qu'elles offrent par exemple en termes de coût, induit chez les conducteurs un processus de réflexion sur ses activités de déplacement. Il va devoir repenser ses activités (déplacement, planification...) et la manière dont il réalise l'activité (style de conduite) afin de les amener à évoluer. La voiture électrique va donc représenter un agent transformateur des activités de déplacements. La planification et la conduite vont permettre de réaliser des activités de déplacement plus économes, plus sûres avec une vigilance accrue et plus efficientes en termes de consommation d'énergie. Ces comportements sont positivement reproduits à l'usage des voitures thermiques. En vue de participer au maintien de l'usage de la VE à long terme chez de nouveaux conducteurs, le développement de ces comportements pourrait être favorisé. Il pourrait être pertinent d'accompagner ce changement technologique (passer d'une voiture thermique à une électrique), d'aider au développement de nouveaux usages, de nouvelles pratiques de conduite. Cela pourrait passer par la création de groupe de partage de «bonnes pratiques », de communauté de possesseur de VE plus ou moins formel (forum internet, rencontre entre possesseurs de $V E . .$.$) . L'usage des VE engendre égalent des réflexions chez les conducteurs concernant$ leurs rapports aux autres usagers de la route, que ce soit les autres conducteurs ou les piétons. Les rapports interindividuels sont également modifiés avec la charge extérieure au domicile, nécessitant de repenser et créer de nouvelles modalités et règles d'interactions. Cela leur permet de maintenir une certaine stabilité dans le système 
d'activité sociodomestique, ainsi qu'une continuité dans leurs pratiques et activités dépendantes des déplacements. Les groupes de partages autour des «bonnes pratiques » pourraient faciliter la création de nouvelles règles régissant les différentes $\mathrm{SA}$ auquel appartiennent les conducteurs de VE. Notamment la création de règles implicites ou modalités d'interactions régissant la charge au domicile d'autrui (pouvant être considérée comme inappropriée) ou les rapports avec les autres usagers de la route qui peuvent être problématiques. Cet accompagnement au changement technologique favoriserait cette tendance déjà majoritairement positive dans la manière dont l'usage d'une VE impacte le quotidien. Cela tend à converger vers une acceptation située positive de la voiture électrique.

\section{BIBLIOGRAPHIE}

Allen, T.M., Lunenfeld, H., \& Alexander, G.J. (1971). Driver information need. Highway Research Board, 36(7), 715-729.

Bardin, L. (2007). L'analyse de contenu. Paris : PUF.

Bobillier-Chaumon, M.-E. (2013). Conditions d'usage et facteurs d'acceptation des technologies dans l'activité : questions et perspectives pour la psychologie du travail. Habilitation à Diriger des Recherches, Université Pierre-Mendès-France, Grenoble.

Bobillier-Chaumon, M.-E. (2016). Acceptation située des TIC dans et par l'activité : premiers étayages pour une clinique de l'usage. Psychologie du Travail et des Organisations, 22(1), 4-21.

Bobillier-Chaumon, M.-E., Cuvillier, B., Durif-Bruckert, C., Cros, F., Vanhille, M., \& Bekkadja, S. (2014). Concevoir une technologie ambiante pour le maintien à domicile : une démarche prospective par la prise en compte des systèmes d'activité. Le Travail humain, 77(1), 39-62.

Bobillier-Chaumon, M.-E., Dubois, M., \& Retour, D. (2006). L'acceptation des nouvelles technologies d'information : le cas des systèmes d'information en milieu bancaire. Psychologie du Travail et des Organisations, 12(4), 247-262.

Bonneau, C. (2010). Conceptualiser l'articulation technologie-organisation dans une perspective communicationnelle : entretien avec Carole Groleau. COMMposite, 13(1), 86-110.

Cahour, B. (2010). Émotion, affects et confort comme nouveau déterminant de l'activité et de l'usage. In G. Vallery, M.-C. Le Port, \& M. Zouinar (Eds.), Ergonomie, conception de produits et de services médiatisés (pp. 273-305). Paris : PUF.

Cahour, B., Brassac, C., Vermersch, P., Bouraouis, J.-L., Pachoud, B., \& Salembier, P. (2007). Étude de l'expérience du sujet pour l'évaluation de nouvelles technologies : l'exemple d'une communication médiée. Revue d'Anthropologie des Connaissances, 1(1), 85-120.

Carroll, J., Howard, S., Peck, J., \& Murphy, J. (2003). From adoption to use : the process of appropriating a mobile phone. Australasian Journal of Information Systems, 10(2), 38-48.

Clot, Y., \& Leplat, J. (2005). La méthode clinique en ergonomie et en psychologie du travail. Le Travail Humain, 68(4), 289-316. 
Dubuisson-Quellier, S., \& Plessz, M. (2013). La théorie des pratiques : quels apports pour l'étude sociologique de la consommation? Sociologie, 4(4).

Engeström, Y. (1987). Learning by expanding : An activity- theoretical approach to developmental research. Helsinki : Orienta-Kosultit.

Flanagan, J.C. (1954). The critical incident technique. Psychological Bulletin, 51(4), 327-358.

Haddon, L. (2011). Domestication Analysis, Objects of Study, and the Centrality of Technologies in Everyday Life. Canadian Journal of Communication, 36(2), 311-323.

Kaufmann, V. (1999). Mobilité et vie quotidienne : synthèse et questions de recherche. (Série synthèses et recherches No. 48) (p. 63). INRETS.

Kuuti, K. (1996). Activity theory as a potential framework for human-computer interaction research. In B. Nardi (Ed.), Context and consciousness : Activity theory and human-computer interaction (pp. 17-44). Cambridge, MA : MIT Press.

Lazarus, R.S., \& Folkman, S. (1984). Stress, appraisal, and coping. NY : Springer.

Licoppe, C. (2008). Dans le « carré de l'activité » : perspectives internationales sur le travail et l'activité. Sociologie du Travail, 50(3), 287-302.

Ling, R. (2004). The adoption, use and social consequences of mobile communication. Telektronikk, 100(3), 69-81.

Mallein, P., \& Toussaint, Y. (1994). L'intégration sociale des technologies d'information et de communications : une sociologie des usages. TIS, 6(4), 115-335.

Millerand, F. (1999). Usages des NTIC : les approches de la diffusion, de l'innovation et de l'appropriation (2e partie). comMposite, 98(1).

Nguyen, C. (2013). Du véhicule thermique au véhicule électrique : pratiques instrumentées et vécus de l'autonomie restreinte. Thèse de doctorat, Télécom ParisTech, Paris.

Nogry, S., Wagner, C., \& Decortis, F. (2015). Appropriation d'une classe mobile à l'école primaire (Research Report). Laboratoire Paragraphe, Université Paris 8, Université Cergy-Pontoise.

Orlikowski, W.J. (1991). The Duality of Technology : Rethinking the Concept of Technology in Organizations. Organization Science, 3(3), 398-427.

Proulx, S. (2002). Trajectoires d'usages des technologies de communication : les formes d'appropriation d'une culture numérique comme enjeu d'une société du savoir. Annales des Télécommunications, 57(3-4), 180-189.

Rabardel, P. (1995). Les hommes et les technologies. Une approche cognitive des instruments contemporains. Paris : Armand Colin.

Reckwitz, A. (2002). Toward a Theory of Social Practices : A Development in Culturalist Theorizing. European Journal of Social Theory, 5(2), 243-263.

Relieu, M., Salembier, P., \& Theureau, J. (2004). Introduction au numéro spécial « Activité et Action/ Cognition Située ». Activités, 1(2), 1-10.

Sahar, A. (2009). Self-reported driving behaviors as a function of trait anxiety. Accident Analysis and Prevention, 41, 241-245.

Schatzki, T.R. (1996). Social Practices : A Wittgensteinian Approach to Human Activity and the Social. Cambridge : Cambridge University Press. 
Shove, E., Pantzar, R., \& Watson, M. (2012). The Dynamics of Social Practice. Everyday Life and how it Changes. London : Sage.

Silverstone, R., \& Haddon, L. (1996). Design and the Domestication of ICTs : Technical Change and Everyday Life. In R. Silverstone \& R. Mansell (Eds.), Communication by Design. The Politics of Information and Communication Technologies (pp. 44-74).Oxford : Oxford University Press.

Smokers, R., \& Kampman, B. (2006). Energy Efficiency in the Transport Sector - Discussion paper prepared for the PEEREA Working Group on Energy Efficiency and Related Environmental Aspects (Report). Delft, Netherlands : CE, Delft.

Stephens, A.N., \& Groegr, J. A. (2009). Situational specificity of trait influences on drivers evaluations and driving behavior. Transportation Research Part F, 12, 29-39.

Suchman, L. A. (1987). Plans and situated actions : the problem of human-machine communication (Vols. 1-1). Cambridge : Cambridge University Press.

Theureau, J. (2004). L'hypothèse de la cognition (ou action) située et la tradition d'analyse du travail de l'ergonomie de langue francaise. Activités, 1(2), 11-25.

Vermersch, P. (1994). L'entretien d'explicitation. Issy-Les-Moulineaux : ESF éditeur.

Vygotsky, L. (1985). Pensée et langage. Paris : Éditions sociales.

Wellings, T., Binnersley, J., Robertson, D., \& Khan, T. (2011). Human machine interfaces in low carbon vehicles: Market trends and user issues. (Report No. Document No. HMI 2.1). Retrieved from http:// www2.warwick.ac.uk/fac/sci/wmg/research/lcvtp/news/hevc11/ ws13_hmi_in_lcvs_market_analysis_and_user_issues_v2_1.pdf

\section{NOTES}

1. http://www.developpement-durable.gouv.fr/IMG/pdf/Chiffres_cles_du_transport_2015.pdf

2. http://www.developpement-durable.gouv.fr/Transports,34304.html

3. Agence de l'environnement et de la maîtrise de l'énergie

4. http://www.automobile-propre.com/dossiers/voitures-electriques/chiffres-venteimmatriculations-france/\#Ventes_de_voitures_electriques_en_2015

5. Art. R412-34 du Code de la route.

6. Art. R.412-37 à R.412-39 du Code de la route.

\section{RÉSUMÉS}

L'étude de l'acceptation de la voiture électrique (VE) nous montre comment cet artefact technique vient transformer les activités socio-domestiques dans un contexte d'usage réel. Des données issues d'entretiens d'explicitation et d'incidents critiques, réalisés auprès de possesseurs/conducteurs de VE $(\mathrm{n}=9)$ montrent que les spécificités de la VE vont amener les conducteurs à repenser leurs pratiques de déplacement et de régulation de leur système d'activité. Ainsi pour faire face à son autonomie limitée, ils développent un certain nombre de stratégies telles que des activités de planification et des comportements d'éco-conduite. De 
même, les conducteurs affirment qu'ils vont accroitre leur vigilance au volant en réponse à la perception de la dangerosité de l'absence de bruit. Ces ajustements vont participer à la genèse instrumentale de la VE et donc à son appropriation. Les différents systèmes d'activité auxquels appartient le conducteur vont également être affectés. Les règles régissant les déplacements du foyer vont ainsi évoluer, tout comme celles régissant les rapports avec autrui (entourage, usagers de la route, professionnels de l'automobile).

The study of the acceptance of the electric car (EC), show how this technical artefact transforms the domestic activities in a real use context. Data from individual explicit interviews with electric car drivers/owners ( $n=9)$ show that the specificities of EC are going to lead the drivers to rethink their driving practices and the regulation of their activity system. To cope with its limited autonomy, they develop strategies such as planning activities and eco-driving behaviors. Besides, drivers are going to increase their vigilance in response to the perception of the dangerousness of the engine noise absence. These adjustments will be part of the instrumental genesis of the electric car and of its appropriation. The rules regulating household trips are going to evolve, as well as those regulating relationships with social others (entourage, road users, automobile professionals).

\section{INDEX}

Mots-clés : voiture électrique, activité domestique, acceptation, appropriation, système d'activité

Keywords : electric car, domestic activity, acceptance, appropriation, activity system

\section{AUTEURS}

\section{LÉNAÏC POUPON}

Université Lyon 2 - Laboratoire GRePS (EA 4163). lenaic.poupon@gmail.com

\section{CHRYSTÈLE PHILIPPS-BERTIN}

Université Lyon, IFSTTAR, AME, LTE, Lyon. chrystele.philipps-bertin@ifsttar.fr

\section{MARC-ÉRIC BOBILLIER CHAUMON}

Université Lyon 2 - Laboratoire GRePS (EA 4163). marc-eric.bobillier-chaumon@univ-lyon2.fr

\section{NIKOS KALAMPALIKIS}

Université Lyon 2 - Laboratoire GRePS (EA 4163). nikos.kalampalikis@univ-lyon2.fr 